\title{
Renewal theory with fat tailed distributed sojourn times: typical versus rare
}

\author{
Wanli Wang ${ }^{1,2}$, Johannes H. P. Schulz ${ }^{2}$, Weihua Deng ${ }^{1}$, and Eli Barkai ${ }^{2}$ \\ ${ }^{1}$ School of Mathematics and Statistics, \\ Gansu Key Laboratory of Applied Mathematics and Complex Systems, \\ Lanzhou University, Lanzhou 730000, P.R. China \\ ${ }^{2}$ Department of Physics, \\ Institute of Nanotechnology and Advanced Materials, \\ Bar-Ilan University, Ramat-Gan 52900, Israel
}

(Dated: October 26, 2018)

\begin{abstract}
Renewal processes with heavy-tailed power law distributed sojourn times are commonly encountered in physical modelling and so typical fluctuations of observables of interest have been investigated in detail. To describe rare events the rate function approach from large deviation theory does not hold and new tools must be considered. Here we investigate the large deviations of the number of renewals, the forward and backward recurrence time, the occupation time, and the time interval straddling the observation time. We show how non-normalized densities describe these rare fluctuations, and how moments of certain observables are obtained from these limiting laws. Numerical simulations illustrate our results showing the deviations from arcsine, Dynkin, Darling-Kac, Lévy and Lamperti laws.
\end{abstract}

PACS numbers: 02. 50. -r, 05. 20. -y, 05. 40. -a

\section{INTRODUCTION}

Renewal processes [1-6] are simple stochastic models for events that occur on the time axis when the time intervals between events are independent and identically distributed (IID) random variables. This idealised approach has many applications, ranging from the analysis of photon arrival times to queuing theory. In some models the sojourn time probability density function (PDF) has fat tails, and this leads to fractal time renewal processes. In the case when the variance of the sojourn time diverges, we have deviations from the normal central limit theorem and/or the law of large numbers. Such fat tailed processes are observed in many systems, ranging from blinking quantum dots [7], to diffusion of particles in polymer networks [8], or diffusion of particles on the membrane of cell [9] to name a few. In these systems the renewal process is triggering jumps in intensity or in space. The continuous time random walk model [10], the annealed trap model, the zero crossing of Brownian motion, the velocity zero crossing of cold atoms diffusing in momentum space [11], are all well known models which use this popular renewal approach (see however $[12,13])$. Heavy tailed renewal theory is also used in the context of localization in random waveguides [14]. The number of renewals, under certain conditions, is described by Lévy statistics, and the fluctuations in these processes are large. Hence it is important to explore the rare events or the far tails of the distributions of observables of interest. As mentioned in $[15,16]$ the large deviation principle, with its characteristic exponential decay of large fluctuations, does not describe this case, and instead the big jump principle [17] is used to evaluate the rare events in Lévy type of processes.

The main statistical tool describing observables of in- terest are non-normalised states, which are limiting laws with which we may obtain statistical information on the system, including for example the variance, which in usual circumstances is the way we measure fluctuations. These non-normalised states were previously investigated, in the context of Lévy walks [18], spatial diffusion of cold atoms [19], and very recently for BoltzmannGibbs states when the underlying partition function of the system diverges [20]. These functions describing the statistical behavior of the system are sometimes called infinite densities or infinite covariant densities, and they appear constantly in infinite ergodic theory [21].

Our goal in this paper is to investigate the statistics of rare events in renewal theory. Consider for example a non-biased ordinary random walk on the integers. The spatial jump process is Markovian hence the zero crossing, where the zero is the origin, is a renewal process. Here like Brownian motion, the waiting time PDF between the zero crossings is fat tailed, in such a way that the mean return time diverges. The distribution of the occupation time $0<T^{+}<t$, namely the time the random walker spends in the positive domain is well investigated [1, 22]. Naively one would expect that when the measurement time $t$ is long the particle will spend half of its time to the right of the origin. Instead one finds that this is the least likely scenario, and the PDF of the properly scaled occupation time reads

$$
\lim _{t \rightarrow \infty} f_{T^{+} / t}(x)=\frac{1}{\pi \sqrt{x(1-x)}} .
$$

Here and all along this manuscript the subscript denotes the observable of interest, e.g. we consider the PDF of $T^{+} / t$ which attains values $0<x<1$. This arcsine law, which describes also other features of Brownian motion [23-25], exhibits divergences on $x \rightarrow 0$ or $x \rightarrow 1$. Here a 
particular scaling of $T^{+} \propto t$ is considered. However, in cases studied below we show that other limiting laws are found when a second time scale is considered and these may modify the statistical properties of the occupation time when $T^{+}$is either very small or very large. This in turn influences the anticipated blow up of the arcsine law at its extremes. Notice that here the least likely event, at least according to this law is the case $x=1 / 2$, so our theory is not dealing with corrections to the least likely event, but rather corrections to the most likely events. This is because of the fat tailed waiting times, which make the discussion of deviations from familiar limiting laws a case study in its own right. While the theory deals with most likely events, from the sampling point of view these are still rare, as the probability of finding the occupation time in a small interval close to the extremes of the arcsine law is still small.

The organization of the paper is as follows. In section II, we outline the model and give the necessary definitions. The behavior of the probability of observing $N$ renewals in the interval $(0, t), p_{N}(t)$ is analyzed in section III. In sections IV, V and VI, the densities of the forward and backward recurrence time, and the time interval straddling $t$, denoted $F, B$ and $Z$ respectively, are derived. In order to see the effects of the typical fluctuations and large deviations, the fractional moments, e.g., $\left\langle F^{q}\right\rangle$, are considered and bi-fractal behavior is found. In section VII, the behavior of the occupation time $T^{+}$is studied. In the final section, we conclude the paper with some discussions. All along our work we demonstrate our results with numerical experiments and compare between the statistical laws describing typical fluctuations to those found here for the rare events.

\section{MODEL}

Renewal process, an idealized stochastic model for events that occur randomly in time, has a very rich and interesting mathematical structure and can be used as a foundation for building more realistic models [26, 27]. As mentioned, the basic mathematical assumption is that the time between the events are IID random variables. Moreover, renewal processes are often found embedded in other stochastic processes, most notably Markov chains.

Now, we briefly outline the main ingredients of the renewal process [1]. It is defined as follows: events occur at the random epochs of time $t_{1}, t_{2}, \ldots, t_{N}, \ldots$, from some time origin $t=0$. When the time intervals between events, $\tau_{1}=t_{1}, \tau_{2}=t_{2}-t_{1}, \ldots, \tau_{N}=t_{N}-t_{N-1}$, $\ldots$, are IID random variables with a common $\operatorname{PDF} \phi(\tau)$, the process thus formed is a renewal process (see the top panel of the Fig. 1). We further consider the alternating renewal process $I(t)$ in which the process alternates between + and - states. A classical example is a Brownian motion $x(t)$ in dimension one, where we denote state + with $x(t)>0$ and state - for $x(t)<0$. Generically, we imagine that a device, over time, alternates between on and off states, like a blinking dot [7, 28]. Here we suppose the process starts in + state and stays in that state for a period of time $\tau_{1}$, then goes to - state and remains for time $\tau_{2}$; see bottom panel of the Fig. 1. Clearly, it is natural to discuss the total time in state + or.$- T^{+}$and $T^{-}$are called the occupation times in the + and - state, respectively and $T^{+}+T^{-}=t$. For Brownian motion, $\phi(\tau) \sim \tau^{-3 / 2}$ and the distribution of time in state + is the well known arcsine law.

Motivated by previous studies of complex systems, we consider here PDFs with power law tails, i.e., for large $\tau$

$$
\phi(\tau) \sim \frac{\alpha \tau_{0}^{\alpha}}{\tau^{1+\alpha}} .
$$

In this case, the first moment of $\phi(\tau)$ is divergent for $0<\alpha<1$. Here the index $\alpha>0$ and $\tau_{0}$ is a time scale. As we show below, the full form of $\phi(\tau)$ is of importance for the study of the large fluctuations. An example is the fat tailed PDF [10]

$$
\phi(\tau)= \begin{cases}0, & \tau<\tau_{0} \\ \alpha \frac{\tau_{0}^{\alpha}}{\tau^{1+\alpha}}, & \tau>\tau_{0} .\end{cases}
$$

Using the Tauberian theorem [29], in Laplace space

$$
\widehat{\phi}(s) \sim 1-b_{\alpha} s^{\alpha}
$$

for small $s$, where $s$ is conjugate to $\tau, b_{\alpha}=\tau_{0}^{\alpha}|\Gamma(1-\alpha)|$, and $0<\alpha<1$. In order to simplify the expression, we denote $\widehat{\phi}(s)$ as the Laplace transform of $\phi(\tau)$. When $1<\alpha<2$, the first moment $\langle\tau\rangle=\int_{0}^{\infty} \tau \phi(\tau) d \tau$ is finite and the corresponding Laplace form [10] is

$$
\widehat{\phi}(s) \sim 1-\langle\tau\rangle s+b_{\alpha} s^{\alpha}
$$

for small $s$. Notice that $\widehat{\phi}(0)=1$, which means that the PDF is normalized. We would like to further introduce the one sided Lévy distribution $\phi(\tau)=\ell_{\alpha}(\tau)$ with index $\alpha$, which is used in our simulations to generate the process; see Appendix A. In Laplace space, one sided stable Lévy distribution $\phi(\tau)$ is [26]

$$
\int_{0}^{\infty} \exp (-s \tau) \phi(\tau) d \tau=\exp \left(-s^{\alpha}\right)
$$

and the small $s$ expansion is given by $\widehat{\phi}(s) \sim 1-s^{\alpha}$ with $0<\alpha<1$. For specific choices of $\alpha$, the closed form of the $\ell_{\alpha}(\tau)$ is tabulated for example in MATHEMATICA [30]. In particular, a useful special case is $\alpha=1 / 2$

$$
\ell_{1 / 2}(\tau)=\frac{1}{2 \sqrt{\pi}} \tau^{-\frac{3}{2}} \exp \left(-\frac{1}{4 \tau}\right)
$$

It implies that for large $\tau, \ell_{1 / 2}(\tau) \sim \sqrt{4 \pi}^{-1} \tau^{-3 / 2}$ so the first moment of the sojourn time diverges.

In the following we will draw on the research literature given by Godrèche and Luck [1], which is recommended 


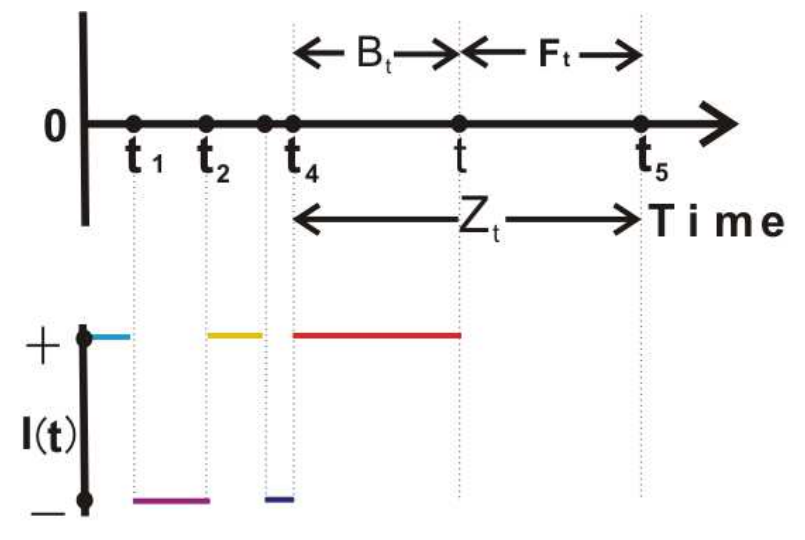

FIG. 1: (color online) Illustration of a renewal process. The $t_{i}$ denotes the time when the $i$-th event occurs. $B_{t}$ and $F_{t}$ present the backward and the forward recurrence time, respectively. In addition, the time interval straddling time $t$ is denoted with $Z_{t}$. The process $I(t)$, an alternating renewal process, is represented in the bottom of the figure. Here we suppose the initial state of the particle is + . We see that the occupation time in the + state is equal to $t_{1}+t_{3}-t_{2}+B_{t}$.

for an introduction. The number of renewal events in the time interval between 0 and time $t$ is

$$
N(t)=\max \left[N, t_{N} \leq t\right]
$$

Then we have the following relation $t_{N}=\tau_{1}+, \ldots,+\tau_{N}$. Now we introduce the forward recurrence time $F_{t}$, the time between $t$ and the next event

$$
F_{t}=t_{N+1}-t ;
$$

see Fig. 1. While the corresponding backward recurrence time, the length between the last event before $t$ and the observation time $t$, is defined by

$$
B_{t}=t-t_{N}
$$

Utilizing the above two equations, we get the time interval straddling time $t$, i.e., $Z_{t}$, which is

$$
Z_{t}=B_{t}+F_{t} .
$$

For simplification, we drop the subscript, denoting the time dependence of the random quantities, from here on.

\section{NUMBER OF RENEWALS BETWEEN 0 AND $t$}

We recap some of the basic results on the statistics of the number of renewal events. The probability of the number of events $N$ up to time $t$ is

$$
p_{N}(t)=\int_{0}^{t} Q_{N}\left(t^{\prime}\right) p_{0}\left(t-t^{\prime}\right) d t^{\prime}
$$

and $Q_{N}\left(t^{\prime}\right)$ is the probability to have an event at time $t^{\prime}$, defined by

$$
Q_{N}(t)=\int_{0}^{t} \phi\left(t^{\prime}\right) Q_{N-1}\left(t-t^{\prime}\right) d t^{\prime}
$$

Here $p_{0}(t)$ is the survival probability

$$
p_{0}(t)=\int_{t}^{\infty} \phi(y) d y
$$

that is, the probability that the waiting time exceeds the observation time $t$. For power law time statistics and large $t$,

$$
p_{0}(t) \sim \frac{b_{\alpha}}{|\Gamma(1-\alpha)| t^{\alpha}} .
$$

Using Eqs. $(8,9)$ and convolution theorem leads to [31]

$$
\widehat{p}_{N}(s)=\widehat{\phi}^{N}(s) \frac{1-\widehat{\phi}(s)}{s}
$$

with $N \geq 0$.

\section{A. Number of renewals between 0 and $t$ with $0<\alpha<1$}

Rewriting Eq. (11), using the convolution theorem of Laplace transform, and performing the inverse Laplace transform with respect to $s$, we get a formal solution

$$
p_{N}(t)=\int_{0}^{t} \mathcal{L}_{\tau}^{-1}\left[\widehat{\phi}^{N}(s)\right] d \tau-\int_{0}^{t} \mathcal{L}_{\tau}^{-1}\left[\widehat{\phi}^{N+1}(s)\right] d \tau,
$$

where $N$ is a discrete random variable and $\mathcal{L}_{\tau}^{-1}\left[\widehat{\phi}^{N}(s)\right]$ means the inverse Laplace transform, from the Laplace space $s$ to real space $\tau$.

Summing the infinite series (summation over $N$ ), the normalization condition $\sum_{N=0}^{\infty} p_{N}(t)=1$ is discovered as expected. We notice that Eq. (12) can be further simplified when $\phi(\tau)$ is one sided Lévy distribution Eq. (5). Then the inverse Laplace transform of Eq. (12) gives

$$
p_{N}(t)=\int_{t /(N+1)^{1 / \alpha}}^{t / N^{1 / \alpha}} \ell_{\alpha}(y) d y .
$$

As usual the large time limit is investigated with the small $s$ behavior of $\widehat{p}_{N}(s)$. Utilizing Eq. (5), the behavior of Eq. (11) in the large $N$ limit and small $s$ is,

$$
\begin{aligned}
\widehat{\bar{p}}_{N}(s) & \rightarrow b_{\alpha} s^{\alpha-1} \exp \left(-N b_{\alpha} s^{\alpha}\right) \\
& =-\frac{1}{N \alpha} \frac{\partial}{\partial s} \exp \left(-N b_{\alpha} s^{\alpha}\right) .
\end{aligned}
$$

Here note that $\int_{0}^{\infty} b_{\alpha} s^{\alpha-1} \exp \left(-N b_{\alpha} s^{\alpha}\right) d N=1 / s$. This means that with this approximation $N$ is treated as a continuous variables, which is fine since in fact we consider a long time limit, and the limiting PDF of 
$N / t^{\alpha}$ is approaching a smooth function. Hence, we have $\bar{p}_{N}(t)$ to denote the continuous approximation. First, using the property of Laplace transform, i.e., $-\int_{0}^{\infty} \exp (-s \tau) \tau f(\tau) d \tau=\frac{\partial}{\partial s} \widehat{f}(s)$, secondly, performing the inverse Laplace transform on the above equation, we find the well known result [1, 21, 32]

$$
\bar{p}_{N}(t) \sim \frac{t}{\alpha N^{1+1 / \alpha} b_{\alpha}^{1 / \alpha}} \ell_{\alpha}\left(\frac{t}{\left(N b_{\alpha}\right)^{1 / \alpha}}\right) .
$$

Eq. (14) is customarily called the inverse Lévy PDF. Furthermore, using $\widehat{\bar{p}}_{N}(s)$, we find that $\widehat{\bar{p}}_{u}(t)$ can be expressed as Mittag-Leffler probability density

$$
\widehat{\bar{p}}_{u}(t)=t^{\alpha-1} E_{\alpha, \alpha}\left(-u t^{\alpha} / b_{\alpha}\right),
$$

where $\widehat{\bar{p}}_{u}(t)$ is the Laplace transform of $\bar{p}_{N}(t)$ with respect to $N$ and a two-parameter function of the MittagLeffler type is defined by the series expansion [33]

$$
E_{\gamma, \nu}(z)=\sum_{n=0}^{\infty} \frac{z^{n}}{\Gamma(\gamma n+\nu)}
$$

with $\gamma>0$ and $\nu>0$. Eq. (14) describes statistics of functionals of certain Markovian processes, according to the Darling-Kac theorem. It was also investigated in the context of infinite ergodic theory [21] and continuous time random walks.

The well known limit theorem Eq. (14) is valid when $N$ and $t$ are large and the ratio $N / t^{\alpha}$ is kept fixed. Now we consider rare events when $N$ is kept fixed and finite, say $N \sim 0,1,2,3$ and $t$ is large. Using Eq. (12) we find

$$
\lim _{t \rightarrow \infty} t^{\alpha} p_{N}(t)=\frac{b_{\alpha}}{\Gamma(1-\alpha)} .
$$

Note that $0<p_{N}(t)<1$ is a probability, while $\bar{p}_{N}(t)$ is a PDF. To make a comparison between Eq. (14) and Eq. (15) we plot in Fig. 2, the probability that $N$ is in the interval $\left(0, N_{1}\right)$ versus $N_{1}$ and compare these theoretical predictions to numerical simulations. Integrating Eq. (14) between 0 and $N_{1}$, gives what we call the typical fluctuations. While the result Eq. (15) exhibits a staircase since according to this approximation

$$
\begin{aligned}
\operatorname{Prob}\left(0 \leq N<N_{1}\right) & \sim \sum_{N=0}^{\operatorname{floor}\left[N_{1}\right]} \frac{b_{\alpha}}{\Gamma(1-\alpha) t^{\alpha}} \\
& \sim\left(\operatorname{floor}\left[N_{1}\right]+1\right) p_{0}(t),
\end{aligned}
$$

where floor $[z]$ gives the greatest integer less than or equal to $z$. From Fig. 2 we see that, besides the obvious discreteness of the probability, deviations between the two results can be considered marginal and noninteresting. Luckily this will change in all the examples considered below, as the statistical description of rare events deviates considerably from the known limit theorems of the field.

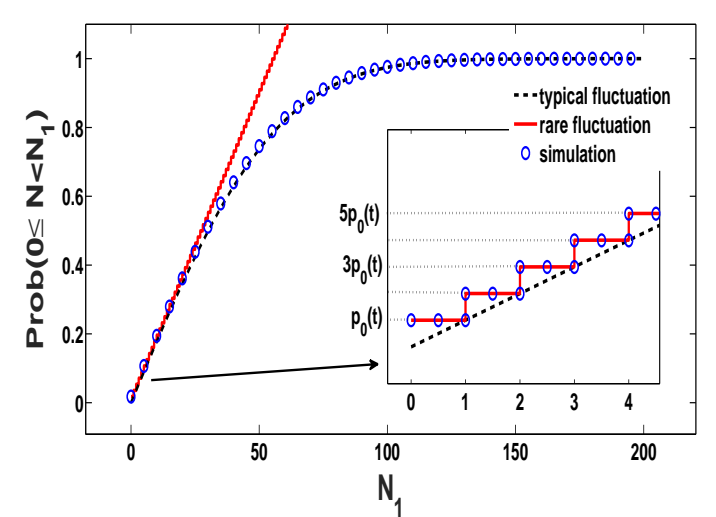

FIG. 2: (color online) Comparison of analytical prediction Eq. (16) (red solid line) for $\operatorname{Prob}\left(0 \leq N<N_{1}\right)$ with typical fluctuations with $\alpha=1 / 2$. We choose $t=1000$, waiting time PDF Eq. (6), and $10^{7}$ trajectories. The typical fluctuations, plotted by dashed (black) lines, are obtained from Eq. (14). The rare fluctuations are given by Eq. (16) and they describe the probability very well for small $N_{1}$ (see inset).

\section{B. Number of renewals between 0 and $t$ with

$$
1<\alpha<2
$$

Based on Eq. (11), we obtain a useful expression

$$
\widehat{\bar{p}}_{u}(s)=\frac{1-\widehat{\phi}(s)}{s} \int_{0}^{\infty} \exp (-u N+N \log (\widehat{\phi}(s))) d N
$$

Here we consider the random variable, $\varepsilon=N-t /\langle\tau\rangle$, and explore its PDF denoted $\bar{p}_{\varepsilon}(t)$. Applying FourierLaplace transform, $\varepsilon \rightarrow k$ and $t \rightarrow s$, the PDF of $\varepsilon$ in Fourier-Laplace space is

$$
\widehat{\bar{p}}_{k}(s)=\frac{1-\widehat{\phi}\left(s+\frac{i k}{\langle\tau\rangle}\right)}{s+\frac{i k}{\langle\tau\rangle}} \frac{1}{-i k-\log \left(\widehat{\phi}\left(s+\frac{i k}{\langle\tau\rangle}\right)\right)} .
$$

First, we consider the limit of small $s$ and small $k$, and the ratio $s /|k|^{\alpha}$ is fixed. As we discuss below this leads to the description of what we call bulk or typical fluctuations, and these are described by standard central limit theorem. Substituting $\widehat{\phi}(s)$ into the above equation and taking inverse Laplace transform

$$
\bar{p}_{k}(t) \sim \exp \left(\frac{b_{\alpha}}{\langle\tau\rangle}\left(\frac{i k}{\langle\tau\rangle}\right)^{\alpha} t\right) .
$$

Fourier inversion of the above equation yields the PDF $\bar{p}_{\varepsilon}(t)$, written in a scaling form [1]

$$
\bar{p}_{\varepsilon}(t) \sim \frac{1}{C_{e v} t^{1 / \alpha}} L_{\alpha, 1}(\xi)
$$

with $C_{e v}=\left(b_{\alpha} /\langle\tau\rangle^{1+\alpha}\right)^{1 / \alpha}$ and $\xi=\varepsilon /\left(C_{e v} t^{1 / \alpha}\right)$; see Fig. 3. We see that for fixed observation time $t$ the parameter $C_{e v}$ measures the PDF's width. Furthermore, 
the function $L_{\alpha, 1}(x)$ is defined by

$$
L_{\alpha, 1}(x)=\frac{1}{2 \pi} \int_{-\infty}^{\infty} \exp (-i k x) \exp \left[(i k)^{\alpha}\right] d k,
$$

where $L_{\alpha, 1}(x)$ is the asymmetric Lévy PDF; see Appendix C. Compared with the one sided Lévy distribution, $L_{\alpha, 1}(x)$ holds two sides with the right hand side decaying rapidly. Moreover, the second moment of $L_{\alpha, 1}(x)$ diverges for $1<\alpha<2$.

As well known the central limit theorem (here of the Lévy form) describes the central part of the distribution, but for finite though large $t$ it does not describe the rare events, i.e., the far tail of the distribution. So far we investigated the typical or bulk statistics and as we showed they are found for $N-t /\langle\tau\rangle \sim t^{1 / \alpha}$. Technically this was obtained using the exact Laplace-Fourier transform, and then searching for a limit where $s$ and $|k|^{\alpha}$ are small their ratio finite, as mentioned. However, it turns out that this limit is not unique. As we now show we can use the exact solution, assume both $k$ and $s$ are small, but their ratio $s /|k|$ finite and obtain a second meaningful solution. This in turn, leads to the description of rare events, i.e., the far tail of the distribution of the random variable $N$. Roughly speaking, in this problem (and similarly all along the paper) we have two scales, one was just obtained and it grows like $t^{1 / \alpha}$, the second (with this example) is $t /\langle\tau\rangle$, as we now show. This means that we have two ways to scale data, one emphasizing the bulk fluctuations (explained already) and the second the rare events.

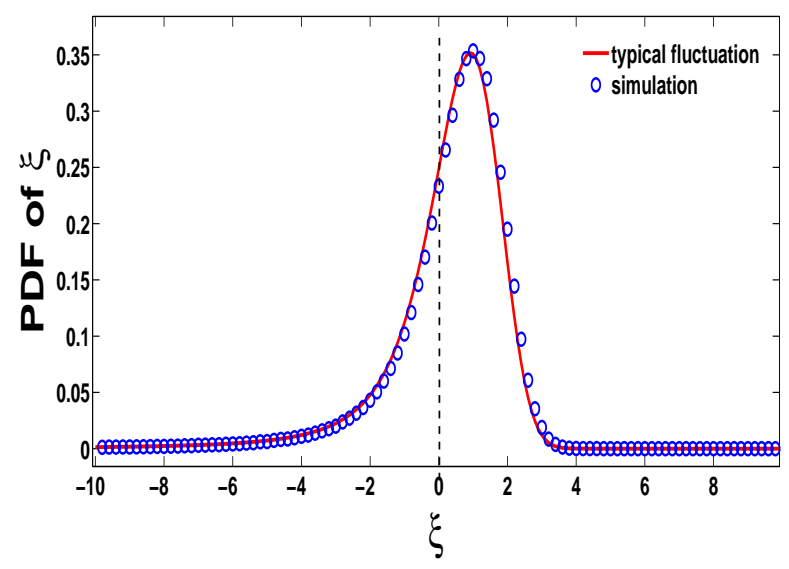

FIG. 3: (color online) Simulation of the number of renewals using the rescaled variable $\xi=(N-t /\langle\tau\rangle) /\left(C_{e v} t^{1 / \alpha}\right)$. We show that the distribution of $\xi$ obtained from numerical simulations, converges to the Lévy density $L_{\alpha, 1}(\xi)$ Eq. (20). This law describes the typical fluctuations, when $N-t /\langle\tau\rangle$ is of the order of $t^{1 / \alpha}$. For simulation we used $t=1000,3 \times 10^{6}$ realizations, $\alpha=1.5$, and $\phi(\tau)$ given in Eq. (2). As the figure shows, the right hand side of $L_{\alpha, 1}(\xi)$ tends toward to zero rapidly.

We now consider a second limiting law capturing the rare events valid when $\varepsilon$ is of the order of $t /\langle\tau\rangle$. From

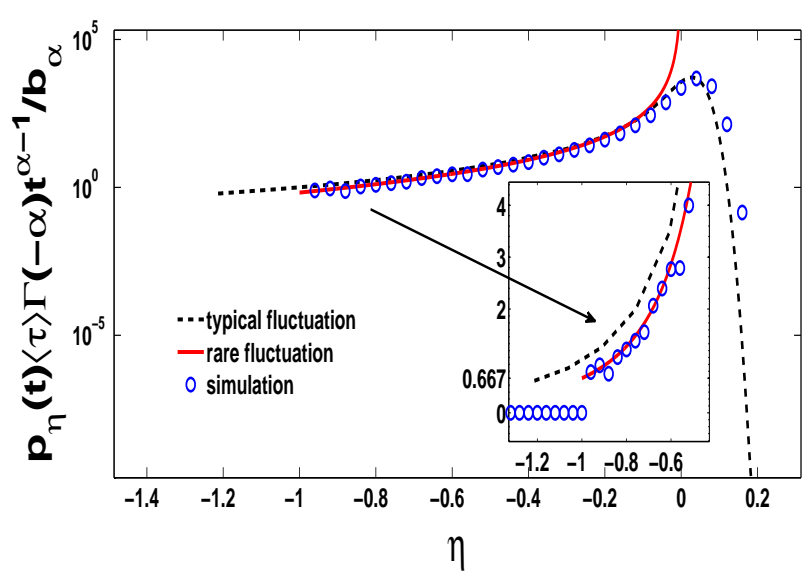

FIG. 4: (color online) Simulation of renewal process with Eq. (2), yields $N$. We show how the scaled PDF of $\eta=$ $(N-t /\langle\tau\rangle) /(t /\langle\tau\rangle)$, obtained from numerical simulations of the renewal process, converges to the non-normalized state Eq. (22). The bulk description Eq. (20) (dashed line) extends to $\eta<-1$, which is certainly not a possibility, and further it is not a valid approximation for $\eta \rightarrow-1$. Here we choose $t=1000, \alpha=1.5$ and $\tau_{0}=0.1$. Deviations from typical fluctuations are clearly illustrated in the inset.

Eq. (18), we have

$$
\widehat{\bar{p}}_{k}(s) \sim \frac{1}{s}-\frac{b_{\alpha}}{\langle\tau\rangle s}\left(s+\frac{i k}{\langle\tau\rangle}\right)^{\alpha-1}+\frac{b_{\alpha}}{\langle\tau\rangle s^{2}}\left(s+\frac{i k}{\langle\tau\rangle}\right)^{\alpha} .
$$

Keep in mind that $s$ and $k$ are small and they are the same order. After performing inverse Fourier-Laplace transform, the asymptotic behavior of $\bar{p}_{\varepsilon}(t)$ is

$$
\bar{p}_{\varepsilon}(t) \sim-\frac{b_{\alpha} \alpha}{\Gamma(1-\alpha)}\left(t(-\varepsilon\langle\tau\rangle)^{-\alpha-1}+\frac{1-\alpha}{\alpha}(-\varepsilon\langle\tau\rangle)^{-\alpha}\right) .
$$

This is the main result of this section. Here the above equation is only valid for negative $\varepsilon$. We see that $\bar{p}_{\varepsilon}(t)$ decays like $t(-\varepsilon)^{-\alpha-1}$ for small negative $\varepsilon$. Moreover, the scaling behavior of $\eta=\varepsilon\langle\tau\rangle / t$ yields

$$
\bar{p}_{\eta}(t) \sim \frac{b_{\alpha}(-\eta)^{-\alpha-1}}{\langle\tau\rangle \Gamma(-\alpha) t^{\alpha-1}}\left(1-\frac{1-\alpha}{\alpha} \eta\right)
$$

with $-1<\eta<0$; see Fig. 4 . It means that $\bar{p}_{\eta}(t)$ decays like $(-\eta)^{-\alpha-1}$ for $\eta \rightarrow 0$, thus $\bar{p}_{\eta}(t)$ is not normalized. Furthermore, for $\eta \rightarrow 0$, the dominating term $(-\eta)^{-1-\alpha}$ matches the left tail of Eq. (20); see Eq. (C3) in Appendix C.

For fixed observation time $t$, the central part of the $\mathrm{PDF} \bar{p}_{\varepsilon}(t)$ is well illustrated by the typical fluctuations Eq. (20). While, its tail is described by Eq. (22), exhibiting the rare fluctuations. In order to discuss the effect of typical fluctuations and large deviations, we further consider the absolute moment of $\varepsilon$ [34], defined by

$$
\left\langle|\varepsilon|^{q}\right\rangle=\int_{-\infty}^{\infty}|\varepsilon|^{q} \bar{p}_{\varepsilon}(t) d \varepsilon
$$


Utilizing Eqs. (20, 22, 23)

$$
\left\langle|\varepsilon|^{q}\right\rangle \sim \begin{cases}\left(C_{e v}\right)^{q} t^{q / \alpha} \int_{-\infty}^{\infty}|z|^{q} L_{\alpha, 1}(z) d z, & q<\alpha \\ \frac{b_{\alpha} q t^{q+1-\alpha}}{|\Gamma(1-\alpha)|\langle\tau\rangle^{q+1}(1+q-\alpha)(q-\alpha)}, & q>\alpha .\end{cases}
$$

Here we use the fact that $\int_{-\infty}^{\infty}|z|^{q} L_{\alpha, 1}(z) d z$ is a finite constant for $q<\alpha$. Note that to derive Eq. (24) we use the non-normalized solution Eq. (21) for $q>\alpha$, indicating that Eq. (21) while not being a probability density, does describe the high order moments. In the particular case $q=2$ (high order moment), we have $\left\langle|\varepsilon|^{2}\right\rangle=\left\langle\left(n-\frac{t}{\langle\tau\rangle}\right)^{2}\right\rangle \sim\left\langle n^{2}\right\rangle-\langle n\rangle^{2} \sim \frac{2 \tau_{0}^{\alpha}}{\langle\tau\rangle^{3}(\alpha-2)(\alpha-3)} t^{3-\alpha}$. While this result is known [1], our work shows that the second moment, in fact any moment of order $q>\alpha$, stems from the non-normalized density describing the rare fluctuations Eq. (21). Other examples of such infinite densities will follow.

Remark 1 From simulations of the number of renewals $N$, Fig. 4, we see deviations from typical results when $\eta>$ 0 . As mentioned, our theory covers the case $\eta<0$, so there is a need to extend the theory further. Note that for $\eta>0$, the typical fluctuations decay rapidly, while power law decay, for intermediate values of $\eta$, is found on the left (see Fig. 4). This intermediate power law behaviors can not continue forever, since $N \geq 0$, and hence when $\eta \approx-1$ a new law emerges, Eq. (22). Possibly the large deviation principle can be used to investigate the case $\eta>0$.

\section{THE FORWARD RECURRENCE TIME}

Several authors investigated the distribution of $F$ both for $F \propto t$, meaning $F$ is of the order of $t$, for $0<\alpha<1$ and also $F \propto t^{0}$ for $\alpha>1$; see Refs. [29, 32, 35]. These works considered the typical fluctuations of $F$, while we focus on the events of large deviations. This means that we consider $F \propto t^{0}$ for $\alpha<1$ and $F \propto t$ for $1<\alpha$. The forward recurrence time is an important topic of many stochastic processes, such as aging continuous time random walk processes (ACTRW) [32, 36], sign renewals of Kardar-Parisi-Zhang Fluctuations [37] and so on. The forward recurrence time, also called the excess time (see schematic Fig. 1), is the time interval between next renewal event and $t$. In ACTRW, we are interested in the time interval that the particle has to wait before next jump if the observation is made at time t. The PDF of the forward recurrence time is related to $Q_{N}(t)$ according to

$$
f_{F}(t, F)=\sum_{N=0}^{\infty} \int_{0}^{t} Q_{N}(\tau) \phi(t-\tau+F) d \tau
$$

see Eq. (B2) in Appendix B. In double Laplace space, the PDF of $F[1]$ is

$$
\widehat{f}_{F}(s, u)=\frac{\widehat{\phi}(u)-\widehat{\phi}(s)}{s-u} \frac{1}{1-\widehat{\phi}(s)} .
$$

Based on the above equation, we will consider its analytic forms and asymptotic ones. In general case, the inversion of Eq. (26) is a function that depends on $F$ and $t$. While, for $\phi(\tau)=\exp (-\tau)$, the above equation can be simplified as $f_{F}(t, F)=\exp (-F)$, which is independent of the observation time $t$. As expected, for this example we do not have an infinite density, neither multi-scaling of moments, since $\exp (-F)$ and more generally thin tailed PDFs, do not have large fluctuations like Lévy statistics.

\section{A. The forward recurrence time with $0<\alpha<1$}

First, we are interested in the case of $F \ll t$. In Laplace space, this corresponds to $s \ll u$. From Eq. (26)

$$
\widehat{f}_{F}(s, u) \sim \frac{1-\widehat{\phi}(u)}{u} \frac{1}{1-\widehat{\phi}(s)} .
$$

We notice that Eq. (27) can be further simplified for a specific $\phi(\tau)$, namely Mittag-Leffler PDF [33, 38]. In order to do so, we consider

$$
\phi(\tau)=\tau^{\alpha-1} E_{\alpha, \alpha}\left(-\tau^{\alpha}\right)
$$

with $0<\alpha<1$. In Laplace space, $\widehat{\phi}(s)$ has the specific form

$$
\widehat{\phi}(s)=\frac{1}{1+s^{\alpha}} .
$$

This distribution can be considered as the positive counterpart of Pakes's generalized Linnik distribution [40] with the PDF having the form $\left(1+s^{\alpha}\right)^{-\beta}, 0<\alpha<2$, $\beta>0$. Plugging Eq. (29) into Eq. (27) leads to

$$
\widehat{f}_{F}(s, u) \sim \frac{u^{\alpha-1}}{1+u^{\alpha}} \frac{1}{s^{\alpha}} .
$$

Taking the the double inverse Laplace transform yields

$$
f_{F}(t, F) \sim \frac{1}{\Gamma(\alpha)} E_{\alpha, 1}\left(-F^{\alpha}\right) t^{\alpha-1}
$$

see Fig. 5. Notice that $E_{\alpha, 1}(0)=1$, so for $t>0$, the PDF of $F$ for $F \rightarrow 0$ gives $f_{F}(t, 0) \sim t^{\alpha-1} / \Gamma(\alpha)$.

More generally, using Eq. (3), we have

$$
\widehat{f}_{F}(s, u) \sim \frac{1-\widehat{\phi}(u)}{u} \frac{1}{b_{\alpha} s^{\alpha}} .
$$

Performing inverse double Laplace transform leads to the main result of this section, and the density describing the large deviations is

$$
f_{F}(t, F) \sim \frac{\int_{F}^{\infty} \phi(y) d y}{\left\langle\tau^{*}\right\rangle},
$$




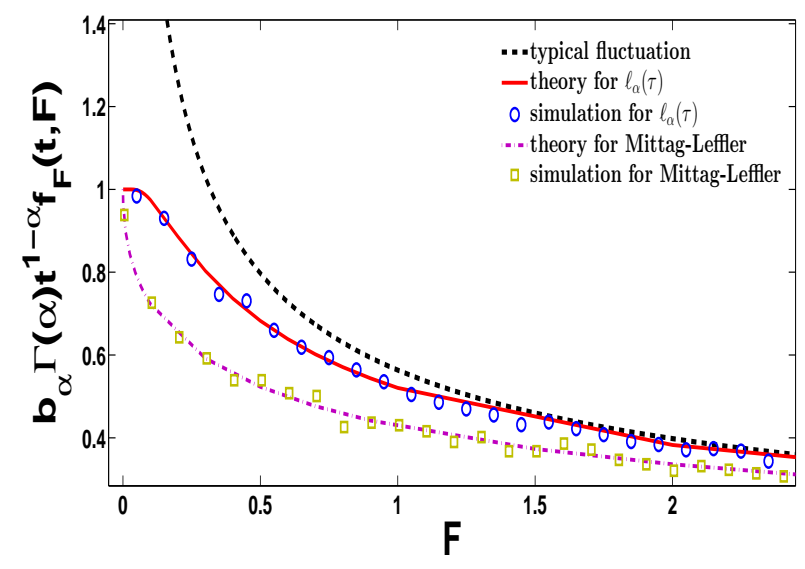

FIG. 5: (color online) The behavior of $f_{F}(t, F)$ for small $F$ with $\alpha=0.5$. The full (red) and the dash-dot (purple) lines describing the large deviations are the analytical results Eqs. (31) and (30), respectively. The dashed (black) line given by Eq. (35) is Dynkin's limit theorem which gives the PDF when $F$ is of the order of $t$, and $t$ is large. Simulations are obtained by averaging $10^{7}$ trajectories with $t=1000$. Note that $b_{\alpha} \Gamma(\alpha) t^{1-\alpha} f_{F}(t, F)$ approaches to one for $F \rightarrow 0$.

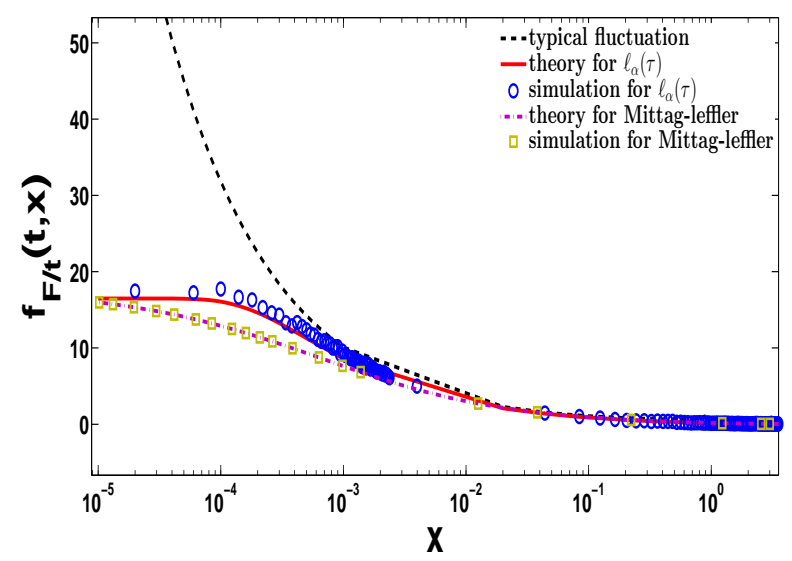

FIG. 6: (color online) The behaviors of $f_{F}(t, F)$ with the scaling variable $x=F / t$ for $\alpha=0.5$ generated by $10^{7}$ trajectories with $t=1000$. The symbols are the simulation results. For $\alpha=0.5$, based on Eq. (35), we get $f_{F / t}(t, x) \sim$ $(\pi(1+x) \sqrt{x})^{-1}$, which is shown by the dashed (black) line. Here we use Eqs. (33) and (34) to predict theoretical results. When $x \rightarrow 0$, the PDF of $F$ depends on particular properties of $\phi(\tau)$, while for large $x$, the details of the PDF become non important, besides the value of $\alpha$.

which exhibits interesting aging effects [32]. Here $\left\langle\tau^{*}\right\rangle$ for large $t$ is equal to $(\Gamma(2-\alpha) \Gamma(1-\alpha)) \int_{0}^{t} \tau^{\prime} \phi\left(\tau^{\prime}\right) d \tau^{\prime} \sim$ $b_{\alpha} \Gamma(\alpha) t^{1-\alpha}$, namely $\left\langle\tau^{*}\right\rangle$ is increasing with measurement time $t$, and for reasons that become clear later we may call it the effective average waiting time (recall the $\langle\tau\rangle$ is a constant only if $\alpha>1)$. The large deviations shows that for large $F$ the forward recurrence time $f_{F}(t, F)$ decays as $F^{-\alpha}$. Furthermore, the integration of Eq. (31) over $F$ diverges since $F^{-\alpha}$ is not integrable for large $F$. Hence Eq. (31) is not a normalised density. For that reason, we may call $f_{F}(t, F)$ in Eq. (31) an infinite density [18], the term infinite means non-normalizable, hence this is certainly not a probability density. Even though $f_{F}(t, F)$ Eq. (31) is not normalized, it is used to obtain certain observables, such as averages of observables integrable with respect to this non-normalized state. Besides, infinite densities play an important role in infinite ergodic theory [41, 42] and intermittent maps [43].

Using Eq. (26), we find a formal solution to the problem

$$
f_{F}(t, F)=\phi(t+F) *_{t} \mathcal{L}_{t}^{-1}\left[\frac{1}{1-\widehat{\phi}(s)}\right] .
$$

where ' $*_{t}$ ' is the Laplace convolution operator with respect to $t$ and the double Laplace transform of the function $f(t+F)$ is

$$
\int_{0}^{\infty} \int_{0}^{\infty} \exp (-s t-u F) f(t+F) d t d F=\frac{\widehat{f}(u)-\widehat{f}(s)}{s-u} .
$$

We further discuss a special choice of $\phi(\tau)$, i.e., $\phi(\tau)=$ $\ell_{\alpha}(\tau)$. After some simple calculations, Eq. (32) gives

$$
\begin{aligned}
f_{F}(t, F)= & \sum_{n=1}^{\infty} \frac{1}{n^{1 / \alpha}} \int_{0}^{t} \ell_{\alpha}(t-\tau+F) \ell_{\alpha}\left(\frac{\tau}{n^{1 / \alpha}}\right) d \tau \\
& +\ell_{\alpha}(t+F) .
\end{aligned}
$$

For Mittag-Leffler waiting time Eq. (28), we obtain

$$
\begin{aligned}
f_{F}(t, F)= & (t+F)^{\alpha-1} E_{\alpha, \alpha}\left(-(t+F)^{\alpha}\right) \\
& +\frac{1}{\Gamma(\alpha)} \int_{0}^{t}(\tau+F)^{\alpha-1}(t-\tau)^{\alpha-1} \\
& \times E_{\alpha, \alpha}\left(-(\tau+F)^{\alpha}\right) d \tau,
\end{aligned}
$$

from which we get the PDF of $x=F / t$ plotted in Fig. 6 .

We now focus on the typical fluctuations, namely the case $F \propto t$ and both are large. This means that $s$ and $u$ are small but of the same order. Plugging Eq. (3) into Eq. (26), then taking double inverse Laplace transform, leading to the normalized solution $[1,35]$

$$
f_{F}(t, F) \sim \frac{\sin (\pi \alpha)}{\pi} \frac{1}{\left(\frac{F}{t}\right)^{\alpha}(t+F)},
$$

which is plotted by the dashed (black) lines in Figs. 5 and 6. The well known solution Eq. (35) describes the typical fluctuations when $F \sim t$.

To summarize, the forward recurrence time shows three distinct behaviors: for $0<F \propto t^{0}$, the infinite density Eq. (31) rules, and only in this range, the PDF of $F$ depends on the behavior of $\phi(\tau)$; for $t^{0} \ll F \ll t$, both Eqs. (31) and (35) are valid and predict $f_{F}(t, F) \sim F^{-\alpha}$; for $F \gg t$, we use Eq. (35) and then $f_{F}(t, F) \sim F^{-\alpha-1}$. Note that for certain observables, for example $B$ and $T^{+}$, when $B, T^{+} \rightarrow t$, their PDFs are also governed by the shape of $\phi(\tau)$; see below. 
B. The forward recurrence time with $1<\alpha<2$

For $F \ll t$, according to Eq. (26)

$$
\widehat{f}_{F}(s, u) \sim \frac{1-\widehat{\phi}(u)}{u\langle\tau\rangle s},
$$

where as mentioned $\langle\tau\rangle$ is finite. This can be finally inverted, yielding the typical fluctuations [1, 29, 44]

$$
f_{F}(t, F) \sim \frac{1}{\langle\tau\rangle} \int_{F}^{\infty} \phi(y) d y .
$$

Since $1<\alpha<2$, Eq. (36) is a normalized PDF and independent of the observation time $t$, which is different from Eq. (31), but they have similar forms. This is the reason why in the previous section we called $\left\langle\tau^{*}\right\rangle$ the effective average waiting time.

Next we discuss the uniform approximation, which is valid for varieties of $F$ and large $t$, namely within uniform approximation, we have the only condition that $t$ is large but the ratio of $F$ and $t$ arbitrary. It can be noticed that Eq. (26) can be arranged into the following formula

$$
\widehat{f}_{F}(s, u)=\frac{\widehat{\phi}(u)-1}{(s-u)(1-\widehat{\phi}(s))}+\frac{1}{s-u} .
$$

For $F \neq t$, we may neglect the second term, then using $1-\widehat{\phi}(s) \sim\langle\tau\rangle s$ and inverting we get

$$
f_{F}(t, F) \simeq \frac{1}{\langle\tau\rangle} \int_{F}^{t+F} \phi(y) d y,
$$

which captures both the infinite density and the bulk fluctuations; see Fig. 7. Here, Eq. (37) is true for large $t$ without considering the relation between $t$ and $F$. If $F \ll t$, Eq. (37) can be approximated by Eq. (36).

For the rare fluctuations, i.e., both $s$ and $u$ are small and comparable, inserting Eq. (4) into Eq. (26), yields

$$
\widehat{f}_{F}(s, u) \sim \frac{1}{s}+\frac{b_{\alpha}}{\langle\tau\rangle} \frac{u^{\alpha}-s^{\alpha}}{(s-u) s} .
$$

For $F>0$, taking the double inverse Laplace transform, we find

$$
f_{F}(t, F) \sim \frac{b_{\alpha}}{|\Gamma(1-\alpha)|\langle\tau\rangle}\left(F^{-\alpha}-(F+t)^{-\alpha}\right),
$$

which is consistent with Eq. (36) for large $F$ and $t \rightarrow \infty$. Besides, for $t \rightarrow \infty, f_{F}(t, F)$ decays as $F^{-\alpha}$ independent of the observation time $t$. On the other hand, if $F \gg t, f_{F}(t, F)$ grows linearly with $t$, namely $f_{F}(t, F) \sim$ $t / F^{-1-\alpha}$. In addition, using the asymptotic behavior of $\phi(F)$, for large $F$ the uniform approximation Eq. (37) reduces to Eq. (38). Still as for other examples in this manuscript, we may use Eq. (38) to calculate a class of high order moments (for example $\alpha>q>\alpha-1$ ), i.e., those moments which are integrable with respect to this infinite density.

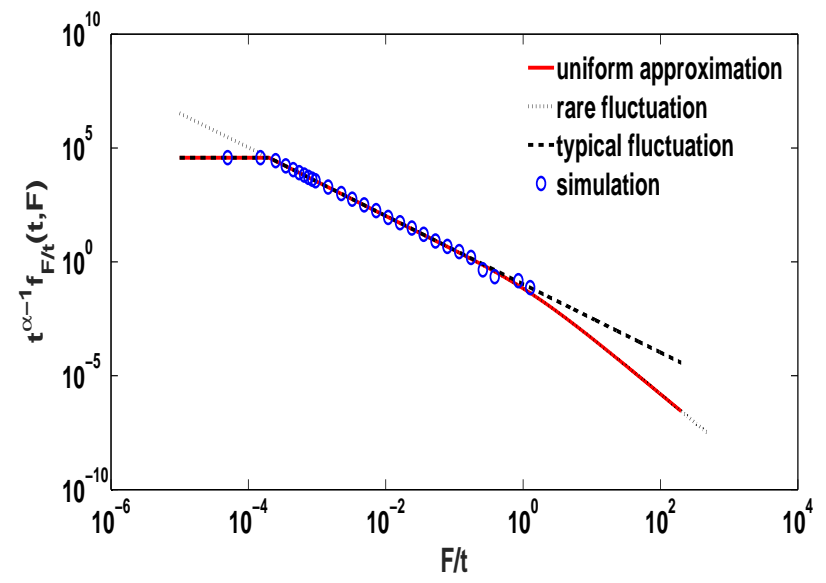

FIG. 7: (color online) The PDF of $x=F / t$ multiplied by $t^{\alpha-1}$ versus $F / t$ generated by $3 \times 10^{6}$ trajectories with Eq. (2). We choose $\alpha=1.5, \tau_{0}=0.1$ and $t=500$. To obtain our theoretical results we used Eqs. (37), (38) and (36). As the figure shows, Eq. (38), describing the large deviations is valid here for large $F / t$, though we experience a sampling problem in simulation (see remark).

Remark 2 For simulations presented in Fig. 7, we use $3 \times 10^{6}$ particles on a standard workstation, taking about 1 day. We see that in this case we do not sample the rare events. In Ref. [18], simulations of the Lévy walk process with $10^{10}$ particles is performed, in order to explore graphically the far tails of the propagator of the Lévy walk. When increasing the number of particles, we will observe rare events, however clearly in our case $3 \times 10^{6}$ realizations are simply not sufficient for meaningful sampling.

With the help of the above equations, now we turn our attention to the fractional moments, defined by

$$
\left\langle F^{q}\right\rangle=\int_{0}^{\infty} F^{q} f_{F}(t, F) d F .
$$

Utilizing Eq. (39) and integration by parts, yields

$$
\left\langle F^{q}\right\rangle \sim\left\{\begin{array}{lr}
\frac{\int_{0}^{\infty} F^{q+1} \phi(F) d F}{(q+1)\langle\tau\rangle}, & q<\alpha-1 ; \\
\frac{b_{\alpha} \Gamma(\alpha-q) \Gamma(1+q) t^{q+1-\alpha}}{\langle\tau\rangle|\Gamma(1-\alpha)| \Gamma(\alpha)(1+q-\alpha)}, & \alpha-1<q<\alpha ; \\
\infty, & q>\alpha .
\end{array}\right.
$$

This is to say, for $q\left\langle\alpha-1,\left\langle F^{q}\right\rangle\right.$ is a constant, namely, it does not depend on the observation time $t$. Moments of order $q<\alpha$ are determined by the known result Eq. (36), which describes typical fluctuations when $F$ is of the order of $t^{0}$. The rare fluctuations, described by Eq. (38), give information to events with $F \propto t$, and this non-normalized density Eq. (38) yields the moments of $\alpha-1<q<\alpha$; see Eq. (D2) in Appendix D. Especially, if $q=1,\langle F\rangle \sim b_{\alpha}(\langle\tau\rangle \Gamma(3-\alpha))^{-1} t^{2-\alpha}$ so in this case the 
mean is determined by the infinite density. When $q>\alpha$, $\left\langle F^{q}\right\rangle$ is divergent. This is expected since the moment of order $q>\alpha$ of $\phi(\tau)$ diverges.

\section{THE BACKWARD RECURRENCE TIME}

Compared with the forward recurrence time, one of the important difference is that $B$ can not be larger than $t$. In some cases, $B$ is called the age at time $t$. Because in the lightbulb lifetime example, it represents the age of the light bulb you find burning at time t. Similar to the derivation of the forward recurrence time

$$
f_{B}(t, B)=\sum_{N=0}^{\infty} \int_{0}^{t} Q_{N}(\tau) \delta(t-\tau-B) \int_{B}^{\infty} \phi(y) d y d \tau .
$$

In Laplace space, let $t \rightarrow s$ and $B \rightarrow u$. Using the convolution theorem of Laplace transform and Eq. (B1), this gives

$$
\widehat{f}_{B}(s, u)=\frac{1-\widehat{\phi}(s+u)}{s+u} \frac{1}{1-\widehat{\phi}(s)},
$$

which was derived in Ref. [1] using a different method.

\section{A. The backward recurrence time with $0<\alpha<1$}

First of all, we study the behaviors of large deviations. For $B \ll t$, i.e., $s \ll u$

$$
\widehat{f}_{B}(s, u) \sim \frac{1-\widehat{\phi}(u)}{u} \frac{1}{1-\widehat{\phi}(s)} .
$$

In the long time limit, i.e., $s \rightarrow 0, \widehat{f}_{B}(s, u) \sim(1-$ $\widehat{\phi}(u)) /\left(b_{\alpha} u s^{\alpha}\right)$. Performing the double inverse Laplace transform with respect to $s$ and $u$, respectively, yields

$$
f_{B}(t, B) \sim \frac{\int_{B}^{\infty} \phi(y) d y}{\left\langle\tau^{*}\right\rangle}
$$

where $\left\langle\tau^{*}\right\rangle$ is defined below Eq. (31). It implies that $\lim _{B \rightarrow 0} f_{B}(t, B)\left\langle\tau^{*}\right\rangle \sim 1$, which is confirmed in Fig. 8 . Furthermore, note that $\int_{0}^{\infty} f_{B}(t, B) d B=\infty$, which means that Eq. (43) is non-normalized.

Now we construct a uniform approximation, which is valid for a wider range of $B$, though $t$ is large. We rewrite Eq. (41) as

$$
\widehat{f}_{B}(s, u)=\left(\frac{1}{s+u}-\frac{\widehat{\phi}(s+u)}{s+u}\right) \frac{1}{1-\widehat{\phi}(s)} .
$$

For simplification, let $\phi(\tau)$ be the one sided Lévy stable distribution Eq. (5). Expanding the above equation, i.e.,

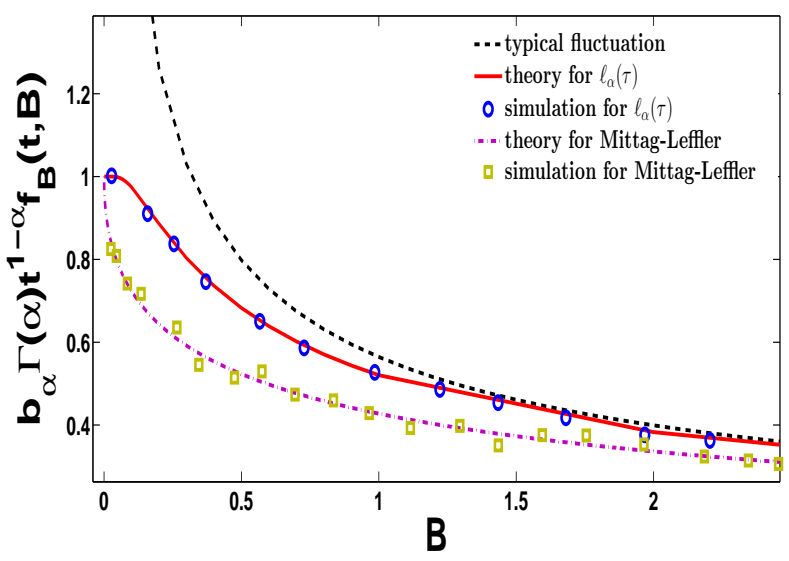

FIG. 8: (color online) The scaled PDF of the backward recurrence time $B$, when $B$ is of the order of unity. The parameters are $t=1000$ and $\alpha=0.5$. The full (red) and dash-dot lines are the analytical result Eq. (43) depicting the large deviations. For the typical result, we used Eq. (50), i.e., $f_{B}(t, B) \sim 1 /(\pi \sqrt{B(t-B)})$. Besides, the symbols are obtained by averaging $10^{7}$ trajectories with one sided Lévy stable distribution Eq. (6) and Mittag-Leffler Eq. (28) time statistics, respectively.

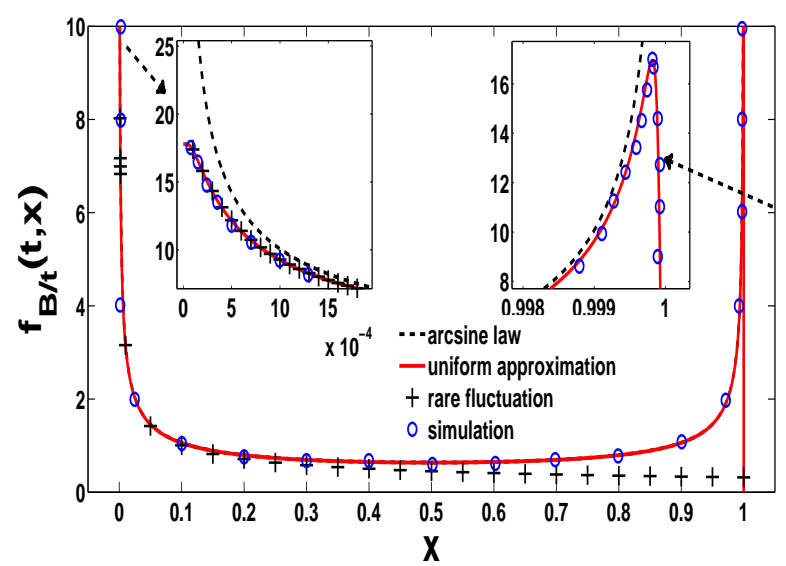

FIG. 9: (color online) The PDF $f_{B / t}(t, x)$ versus $x=B / t$ for a renewal process with $\phi(\tau)$ a one sided Lévy density Eq. (6). Here we choose $t=1000$, and $\alpha=0.5$. The dashed, the full lines and the symbols $(+)$ present the arcsine law Eq. (51), the analytical result Eq. (44), and rare events Eq. (43), respectively. Notice that what the arcsine law predicts here is a symmetric distribution, while our results describing the large deviations exhibit non-symmetry. Furthermore our theory does not blow up at $x \rightarrow 0$ and $x \rightarrow 1$, unlike the arcsine law.

$1 /(1-\widehat{\phi}(s))=\sum_{n=0}^{\infty} \widehat{\phi}^{n}(s)$, and then using the convolu- 


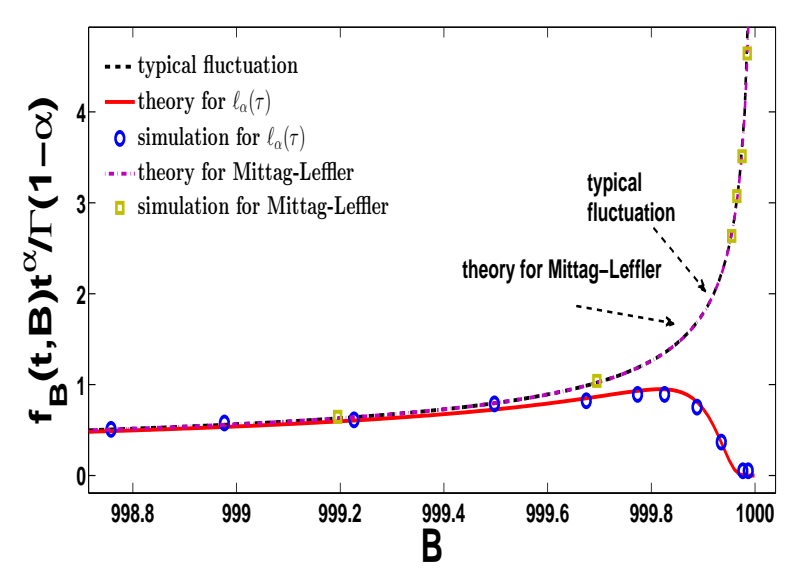

FIG. 10: (color online) The relation between $f_{B}(t, B)$ and $B$ for large $B$. The parameters are $t=1000$ and $\alpha=0.5$. For the typical result we use Eq. (51) and for the large deviations we use Eqs. (48) and (49). The simulations, presented by symbols, are obtained by averaging $10^{7}$ trajectories. It is difficult to distinguish between the typical result and the theoretical result with Mittag-Leffler time statistics, while for the choice of $\phi(\tau)=\ell_{1 / 2}(\tau)$ Eq. (6), the deviations are pronounced.

tion theorem of the Laplace transform

$$
\begin{aligned}
f_{B}(t, B)= & \delta(t-B) \int_{t}^{\infty} \ell_{\alpha}(y) d y+\Theta(t-B) \\
& \times \int_{B}^{\infty} \ell_{\alpha}(y) d y \sum_{n=1}^{\infty} \frac{1}{n^{1 / \alpha}} \ell_{\alpha}\left(\frac{t-B}{n^{1 / \alpha}}\right) ;
\end{aligned}
$$

where $\Theta(z)$ represents the Heaviside theta function [45], which is equal to 0 for $z<0$ and 1 for $z>0$. The $\Theta(t-B)$ in Eq. (44) yields $B \leq t$ as expected. In addition, for $B \ll t$, Eq. (44) reduces to Eq. (43). Note that, for $\alpha=1 / 2$, and comparable $t$ and $B$, Eq. (44) is consistent with the arcsine law, while, let $B$ go to either 0 or $t$ (the extreme cases), the arcsine law does not work anymore; see Fig. 9.

Now we turn our attention to the case of $B \rightarrow t$, using the random variable $\varepsilon=t-B \rightarrow 0$. In Laplace space, the PDF of $\varepsilon$ is

$$
\begin{aligned}
\widehat{f}_{\varepsilon}\left(s, u_{\varepsilon}\right) & =\int_{0}^{\infty} \int_{0}^{\infty} \exp \left(-s t-u_{\varepsilon} \varepsilon\right) f_{\varepsilon}(t, \varepsilon) d t d \varepsilon \\
& =\widehat{f}_{B}\left(s+u_{\varepsilon},-u_{\varepsilon}\right) .
\end{aligned}
$$

According to Eq. (41)

$$
\widehat{f_{\varepsilon}}\left(s, u_{\varepsilon}\right)=\frac{1-\widehat{\phi}(s)}{s} \frac{1}{1-\widehat{\phi}\left(s+u_{\varepsilon}\right)} .
$$

For $s \ll u_{\varepsilon}$, performing the double inverse Laplace transform and using $B=t-\varepsilon$

$$
f_{B}(t, B)=\int_{t}^{\infty} \phi(y) d y \mathcal{L}_{t-B}^{-1}\left[\frac{1}{1-\widehat{\phi}\left(u_{\varepsilon}\right)}\right] .
$$

Let us consider a situation in which $\phi(\tau)$ is the MittagLeffler distribution Eq. (28) with $0<\alpha<1$. Next, plugging Eq. (29) into Eq. (47) yields

$$
f_{B}(t, B)=p_{0}(t) \delta(t-B)+p_{0}(t) \frac{(t-B)^{\alpha-1}}{\Gamma(\alpha)} .
$$

It demonstrates that $t^{\alpha} f_{B}(t, B)$ decays like $(t-B)^{\alpha-1}$. Thus, if $B$ tends to the observation time $t$, we discover an interesting phenomenon that $t^{\alpha} f_{B}(t, B) \rightarrow \infty$, verified in Fig. 10. In general case, Eq. (47) is not easy to calculate in real time exactly, though we use the numerical inversion of Laplace transform by MATLAB. Expanding the above equation, we find

$$
f_{\varepsilon}(t, \varepsilon)=\int_{t}^{\infty} \phi(y) d y\left(\delta(\varepsilon)+\sum_{n=1}^{\infty} \mathcal{L}_{\varepsilon}^{-1}\left[\widehat{\phi}^{n}\left(u_{\varepsilon}\right)\right]\right) .
$$

Consider a specific $\phi(\tau)$, namely one sided Lévy stable distribution

$f_{B}(t, B)=\int_{t}^{\infty} \ell_{\alpha}(y) d y\left(\delta(t-B)+\sum_{n=1}^{\infty} \frac{1}{n^{1 / \alpha}} \ell_{\alpha}\left(\frac{t-B}{n^{1 / \alpha}}\right)\right)$,

which can be used for plotting. To summarize, large deviations are observed for $B \propto t^{0}$ and $B \rightarrow t$, Eq. (43) and Eqs. $(48,49)$ respectively (see Fig. 9), and these are non-symmetric for one sided Lévy distribution. Only when $B \sim t^{0}$, we find a non-normalized density, Eq. (43).

Next we discuss the typical fluctuations when $B \propto t$. Combining Eqs. (3) and (41), yields [1]

$$
f_{B}(t, B) \sim \frac{\sin (\pi \alpha)}{\pi} \frac{1}{B^{\alpha}(t-B)^{1-\alpha}} \Theta(t-B) .
$$

In a particular case $\alpha=1 / 2$, Eq. (50) reduces to the arcsine law $f_{B}(t, B) \sim(\pi \sqrt{B(t-B)})^{-1}$, which is plotted by the dashed (black) line in Figs. 8, 9 and 10. Let $x=B / t$, we get a well known formula [1]

$$
f_{B / t}(x) \sim \frac{\sin (\pi \alpha)}{\pi} \frac{1}{x^{\alpha}(1-x)^{1-\alpha}} \Theta(1-x) .
$$

In particular, for $\alpha=1 / 2$, Eq. (51) reduces to the arcsine law $[1]$ on $[0,1]$, see Fig. 9.

\section{B. The backward recurrence time with $1<\alpha<2$}

We again consider the limit $s \ll u$. Combining Eqs. (4) and (41)

$$
\widehat{f}_{B}(s, u) \sim \frac{1-\widehat{\phi}(u)}{u} \frac{1}{\langle\tau\rangle s},
$$

which, by the double inverse Laplace transform, yields the limiting result [29]

$$
f_{B}(t, B) \sim \frac{\int_{B}^{\infty} \phi(y) d y}{\langle\tau\rangle} .
$$


If $B$ goes to $0, f_{B}(t, B)$ reduces to $1 /\langle\tau\rangle$.

Now we turn our attention to the case when $B \rightarrow t$. According to Eq. (46)

$$
f_{B}(t, B) \sim \int_{t}^{\infty} \phi(y) d y \mathcal{L}_{t-B}^{-1}\left[\frac{1}{1-\widehat{\phi}\left(u_{\epsilon}\right)}\right] .
$$

For power law waiting time statistics, Eq. (52) reduces to

$$
f_{B}(t, B) \sim \frac{b_{\alpha}}{|\Gamma(1-\alpha)| t^{\alpha}} \mathcal{L}_{t-B}^{-1}\left[\frac{1}{1-\widehat{\phi}\left(u_{\epsilon}\right)}\right] .
$$

The inverse Laplace transform gives the limiting law when $B \rightarrow t$.

Let us proceed with the discussion of rare fluctuations. Substituting $\widehat{\phi}(u)$ and $\widehat{\phi}(s)$ into (41), leads to

$$
\widehat{f}_{B}(s, u) \sim \frac{1}{s}-\frac{b_{\alpha}}{\langle\tau\rangle s}(s+u)^{\alpha-1}+\frac{b_{\alpha}}{\langle\tau\rangle} \frac{1}{s^{2-\alpha}},
$$

when $s$ and $u$ are of the same order. By inversion of the above equation

$$
f_{B}(t, B) \sim \frac{b_{\alpha}}{\langle\tau\rangle|\Gamma(1-\alpha)|} B^{-\alpha} \Theta(t-B)
$$

with $B>0$. We see that $f_{B}(t, B)$ blows up at $B \rightarrow 0$ and since $1<\alpha<2$ the solution Eq. (53) is non integrable.

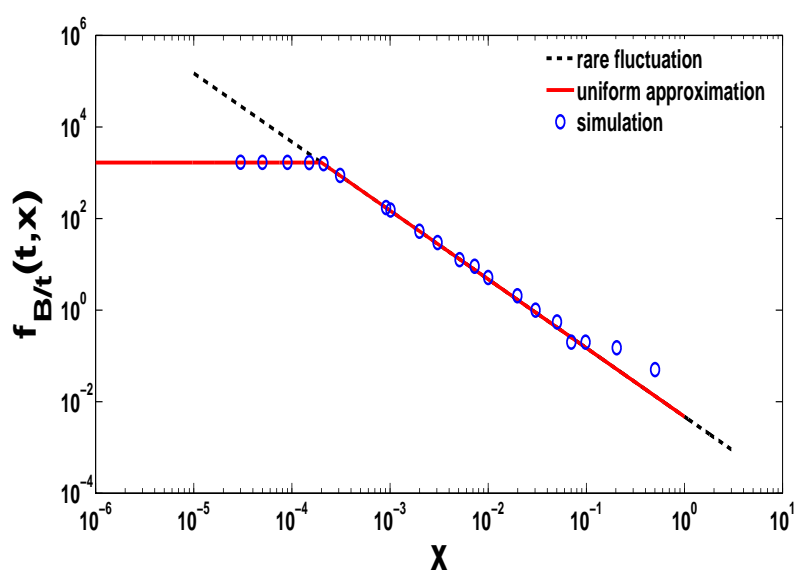

FIG. 11: (color online) The PDF $f_{B / t}(t, B)$ versus the scaling variable $x=B / t$ for the waiting time PDF Eq. (2). The parameters are $t=500, \tau_{0}=0.1$ and $\alpha=1.5$. The solid (red) line is the theory Eq. (54), and the dashed (black) line is Eq. (53), which gives the PDF when $B$ is of the order of $t$, and $t$ is large. The simulations, presented by the symbols, are obtained by averaging $10^{6}$ trajectories.

Next, utilizing Eq. (41), the uniform approximation is

$$
f_{B}(t, B) \sim \frac{1}{\langle\tau\rangle} \Theta(t-B) \int_{B}^{\infty} \phi(y) d y .
$$

Note that $B$ is limited by the observation time $t$. For large $B$, Eq. (54) reduces to Eq. (53).
The corresponding fractional moments are

$$
\left\langle B^{q}\right\rangle \sim \begin{cases}\frac{\int_{0}^{\infty} \phi(B) B^{q+1} d B}{\langle\tau\rangle(q+1)}, & q<\alpha-1 ; \\ \frac{b_{\alpha} t^{q-\alpha+1}}{\langle\tau\rangle|\Gamma(1-\alpha)|(q-\alpha+1)}, & q>\alpha-1 .\end{cases}
$$

Since $B<t$ all moments are finite, unlike the case of the forward recurrence time. The results show that the behaviors of fractional moments are divided into two parts. When $q\left\langle\alpha-1,\left\langle B^{q}\right\rangle\right.$, is determined by the typical fluctuations and it is a constant. The rare fluctuations, described by Eq. (53), give the information on events when $B \propto t$, and this non-normalized limiting law gives the moments of $q>\alpha-1$. The discussion of moments for $\alpha<1$ is given in Appendix E.

\section{THE TIME INTERVAL STRADDLING $t$}

The time interval straddling the observation time $t$ has been studied in Ref. [11, 46-48], where some results about the typical fluctuations are announced and discussed. To consider general initial ensemble in an annealed transit time model [24], one has to consider the time interval straddling time $t$ since the diffusion coefficient is governed by $Z$. Based on the previous result [11], the PDF of $Z$ is given by the double Laplace inversion of

$$
\widehat{f}_{Z}(s, u)=\frac{1}{1-\widehat{\phi}(s)} \frac{\widehat{\phi}(u)-\widehat{\phi}(s+u)}{s},
$$

where $u$ is the Laplace pair of $Z$, and $s$ of $t$. One important feature of $f_{Z}(t, Z)$ is the discontinuity of its derivative at $Z=t$; see below.

\section{A. The time interval straddling $t, 0<\alpha<1$}

Similar to previous sections, we first consider the events of large deviations, namely, $Z$ is of the order of $t^{0}$. Utilizing Eq. (56)

$$
f_{Z}(t, Z) \sim \mathcal{L}_{t}^{-1}\left[\frac{1}{1-\widehat{\phi}(s)}\right] Z \phi(Z)
$$

which gives us an efficient way of calculation for $Z \ll t$. In particular, combining Eqs. (3) and (57), and taking the inverse Laplace transform leads to

$$
f_{Z}(t, Z) \sim \frac{Z \phi(Z)}{\left\langle\tau^{*}\right\rangle},
$$

which is confirmed in Fig. 12. Note that $\left\langle\tau^{*}\right\rangle$ is the same as that defined in Eq. (31). Keep in mind that there is a difference among small $F, B$ and $Z$. For small $Z, f_{Z}(t, Z)$ goes to 0 , while for Eqs. (31) and (43) with $F, B \rightarrow 0$, $f_{F}(t, F)$ and $f_{F}(t, B)$ are equal to $1 /\left\langle\tau^{*}\right\rangle$. In spite of 
these difference, the asymptotic behavior of $f_{Z}(t, Z)$ is consistent with the PDF of the forward recurrence time and the backward one with the increase of $Z$.

We further consider the PDF of $Z$ more exactly. Taking the inverse Laplace transform of Eq. (56) with respect to $u$ and $s$, respectively

$$
\begin{aligned}
f_{Z}(t, Z)= & \phi(Z)\left(\mathcal{L}_{t}^{-1}\left[\frac{1}{s(1-\widehat{\phi}(s))}\right]\right. \\
& \left.-\Theta(t-Z) \mathcal{L}_{t-Z}^{-1}\left[\frac{1}{s(1-\widehat{\phi}(s))}\right]\right) .
\end{aligned}
$$

In particular, for a Mittag-Leffler density Eq. (28), the inversion of Eq. (59) can be further simplified as

$$
\begin{aligned}
f_{Z}(t, Z)= & \frac{\phi(Z)}{\Gamma(1+\alpha)}\left(t^{\alpha}-(t-Z)^{\alpha} \Theta(t-Z)\right) \\
& +\phi(Z)(1-\Theta(t-Z)) .
\end{aligned}
$$

It is interesting to note that Eq. (60) is a uniform approximation for Mittag-Leffler sojourn time. In addition, we find that for $Z \ll t$ Eq. (60) reduces to Eq. (58). On the other hand, when $Z>t$, the above equation yields $f_{Z}(t, Z) \sim t^{\alpha} \phi(Z) / \Gamma(1+\alpha)$. For $t \rightarrow 0$, we see from Eq. (60) that $\lim _{t \rightarrow 0} f_{Z}(t, Z)=\phi(Z)$ as expected.

Let us proceed with the discussion of a general waiting time PDF $\phi(\tau)$. Expanding the term $(1-\widehat{\phi}(s))^{-1}$ of Eq. (59) in powers of $\widehat{\phi}(s)$, and then taking the inverse transform results in

$$
\begin{aligned}
f_{Z}(t, Z) & =\phi(Z) \sum_{n=1}^{\infty}\left(\int_{0}^{t} \mathcal{L}_{\tau}^{-1}\left[\widehat{\phi}^{n}(s)\right] d \tau-\Theta(t-Z)\right. \\
& \left.\times \int_{0}^{t-Z} \mathcal{L}_{\tau}^{-1}\left[\widehat{\phi}^{n}(s)\right] d \tau\right)+\phi(Z)(1-\Theta(t-Z)) .
\end{aligned}
$$

For the one sided Lévy stable distribution

$$
\begin{aligned}
f_{Z}(t, Z)= & \ell_{\alpha}(Z) \sum_{n=1}^{\infty} \frac{1}{n^{1 / \alpha}}\left(\int_{0}^{t} \ell_{\alpha}\left(\frac{\tau}{n^{1 / \alpha}}\right) d \tau\right. \\
& \left.-\Theta(t-Z) \int_{0}^{t-Z} \ell_{\alpha}\left(\frac{\tau}{n^{1 / \alpha}}\right) d \tau\right) \\
& +\ell_{\alpha}(Z)(1-\Theta(t-Z)) .
\end{aligned}
$$

Note that Eq. (62) is valid for all kinds of $t$ and $Z$. In Fig. 13, the scaling behaviors of $x=Z / t$ are displayed. If $Z>t$, Eq. (59) reduces to

$$
f_{Z}(t, Z) \sim \frac{1}{\Gamma(1+\alpha) b_{\alpha}} t^{\alpha} \phi(Z) .
$$

For $s, u$ small and comparable, substituting $\widehat{\phi}(s)$ and $\widehat{\phi}(u)$ into Eq. (56) yields

$$
\widehat{f}_{Z}(s, u) \sim \frac{(s+u)^{\alpha}-u^{\alpha}}{s^{1+\alpha}},
$$

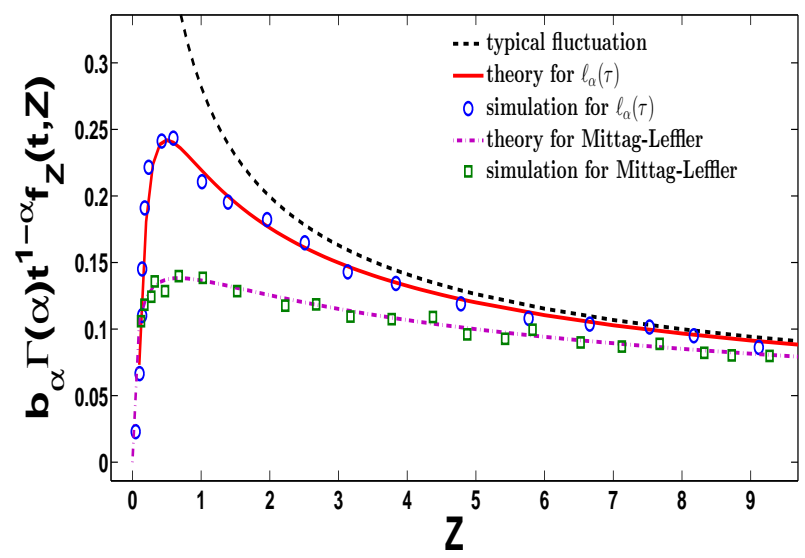

FIG. 12: (color online) Theory and simulations of the statistical behaviors of the rare events of the time interval straddling time $t$, with $\phi(\tau)$ Eqs. (5) and (28) for $t=1000$ and $\alpha=0.5$. The full (red) and dash-dot (purple) lines are theory Eq. (58), showing the large deviations and the corresponding simulation results are presented by symbols obtained by averaging $10^{7}$ trajectories.

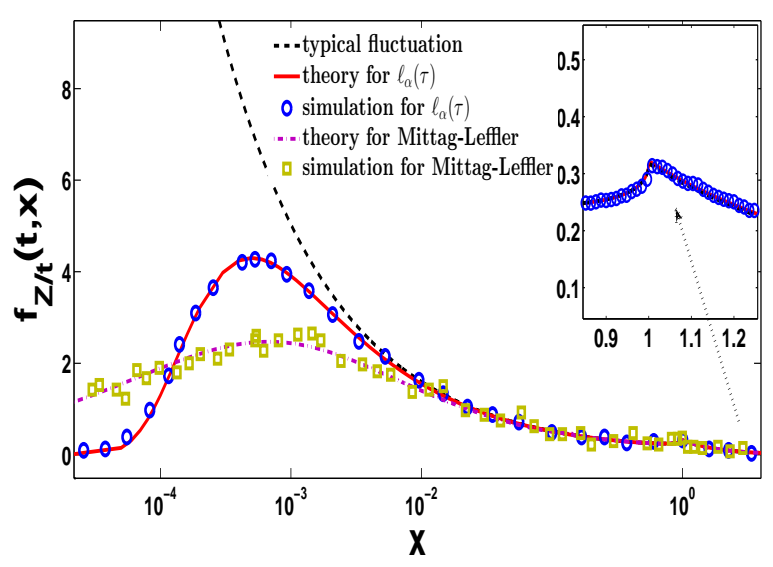

FIG. 13: (color online) The PDF of the rescaled variable $x=Z / t$ with $\alpha=0.5$. The parameters are the same as in Fig. 12. The solid (red) and dash-dot (purple) lines present the theoretical results Eqs. (62) and (60), respectively. Furthermore, the inset demonstrates that the first derivative of $f_{Z}(t, Z)$ is not continuous at $Z=t$.

and then taking the double inverse Laplace transform with respect to $u$ and $s$, gives the typical fluctuations $[11,35]$

$$
f_{Z}(t, Z) \sim \frac{\sin (\pi \alpha)}{\pi} \frac{t^{\alpha}-(t-Z)^{\alpha} \Theta(t-Z)}{Z^{1+\alpha}},
$$

where $\Theta(x)=1$ for $x \geq 0$ and is zero otherwise. 


\section{B. The time interval straddling time $t$ with}

$$
1<\alpha<2
$$

For the typical fluctuations, i.e., $Z \sim t^{0}$. Based on Eq. (56),

$$
f_{Z}(t, Z) \sim \frac{Z \phi(Z)}{\langle\tau\rangle}
$$

see Fig. 14. Note that $f_{Z}(t, Z)$ tends to zero when $Z \rightarrow 0$. We now discuss the rare fluctuations, i.e., $Z$ is of the order of $t$. Plugging Eq. (4) into Eq. (56), then performing the inverse Laplace transform, lead to

$$
f_{Z}(t, Z) \sim \frac{b_{\alpha} Z^{-1-\alpha}}{\langle\tau\rangle \Gamma(-\alpha)}(t-(t-Z) \Theta(t-Z))
$$

with $Z>0$. According to Eq. (66), it gives us another representation of $f_{Z}(t, Z)$, namely

$$
f_{Z}(t, Z) \sim \begin{cases}\frac{b_{\alpha}}{\langle\tau\rangle \Gamma(-\alpha)} Z^{-\alpha}, & Z<t \\ \frac{b_{\alpha}}{\langle\tau\rangle \Gamma(-\alpha)} t Z^{-\alpha-1}, & Z>t .\end{cases}
$$

We now construct a uniform approximation that interpolates between Eqs. (65) and (66). We restart from Eq. (56), but use Eq. (4) only to approximate $1 /(1-\widehat{\phi}(s)) \sim 1 /(\langle\tau\rangle s)$. After performing the double inverse Laplace transform, we arrive at

$$
f_{Z}(t, Z)=\frac{C(t)}{\langle\tau\rangle}(t \phi(Z)-\Theta(t-Z)(t-Z) \phi(Z))
$$

where we have added $C(t)=\langle\tau\rangle /\left(\int_{0}^{t} Z \phi(Z) d Z+\right.$ $\left.\int_{t}^{\infty} t \phi(Z) d Z\right)$ as a normalizing factor, satisfying $\lim _{t \rightarrow \infty} C(t)=1$. In the long time limit, Eq. (67) gives

$$
f_{Z}(t, Z) \sim \frac{t}{\langle\tau\rangle} \phi(Z)
$$

with $Z>t$. It can be seen that Eq. (68) grows linearly with time $t$.

Similar to the calculations of $\left\langle F^{q}\right\rangle$ and $\left\langle B^{q}\right\rangle$

$$
\left\langle Z^{q}\right\rangle \sim\left\{\begin{array}{lr}
\frac{1}{\langle\tau\rangle} \int_{0}^{\infty} Z^{q+1} \phi(Z) d Z, & q<\alpha-1 ; \\
\frac{b_{\alpha} t^{q-\alpha+1}}{\langle\tau\rangle \Gamma(-\alpha)(q-\alpha+1)(\alpha-q)}, & \alpha-1<q<\alpha ; \\
\infty, & \alpha<q .
\end{array}\right.
$$

As expected $\left\langle Z^{0}\right\rangle=1$. Similar to the previous examples, when $\alpha-1<q<\alpha$, the moments $\left\langle Z^{q}\right\rangle$ are obtained from Eq. (66), which is not a normalized PDF. In particular, expanding the right hand side of Eq. (56) to first order in $u$, and taking the inverse Laplace transform, lead to $\langle Z\rangle \sim(\langle\tau\rangle \Gamma(3-\alpha))^{-1} b_{\alpha} \alpha t^{2-\alpha}$, which agrees with Eq. (69).

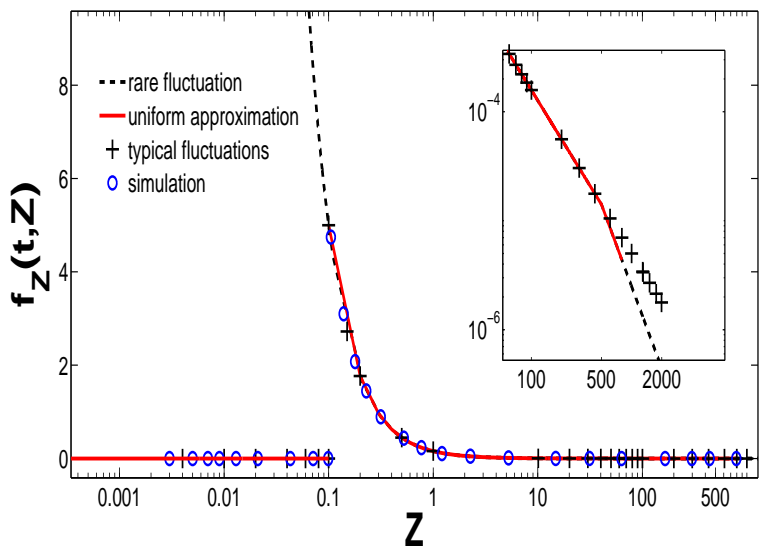

FIG. 14: (color online) The PDF of the straddling time $Z$ versus $Z$ for $\phi(\tau)$ Eq. (2). The parameters are the same as in Fig. 11. The rare fluctuations are given by Eq. (66) (the dashed (black) line), depicting the behaviors when $Z \propto t$. The solid (red) line is the uniform approximation Eq. (67). For the typical fluctuations, we use Eq. (65) which is shown by the symbols $(+)$. When $Z>t$, the rare fluctuations deviate from the typical fluctuations (see the inset).

\section{OCCUPATION TIME}

The occupation time, the time spent by a process in a given subset of the state space during the interval of the observation, is widely investigated in mathematics and physics. It is a useful quantity with a large number of applications, for example the time spent by a one dimensional Brownian motion in half space, the time spent in the bright state for blinking quantum dot models [7, 49], and the time that a spin occupies in a state up [50]. Based on the alternating renewal process, here we focus on the study of the occupation time in the + state. In double Laplace space [1]

$$
\widehat{f}_{T^{+}}(s, u)=\frac{2 s+u}{2 s(s+u)}+\frac{u(\widehat{\phi}(s+u)-\widehat{\phi}(s))}{2 s(s+u)(1-\widehat{\phi}(s+u) \widehat{\phi}(s))}
$$

see the derivation of Eq. (B6) in Appendix B. In this model we start the process in the state up and down with equal probability. Utilizing Eq. (70) and taking $\epsilon=$ $T^{+}-t / 2$, we detect that the $\operatorname{PDF} f_{\epsilon}(t, \epsilon)$ is symmetric with respect to $\epsilon$ for a variety of $\phi(\tau)$. As usual the difficulty is to find the solution in real time, namely find the PDF $f_{T^{+}}\left(t, T^{+}\right)$.

\section{A. Occupation time with $0<\alpha<1$}

We first consider the typical fluctuations, i.e., $T^{+}$is of the order of the measurement time $t$. Substituting Eq. (3) into Eq. (70), and then taking the inverse double 


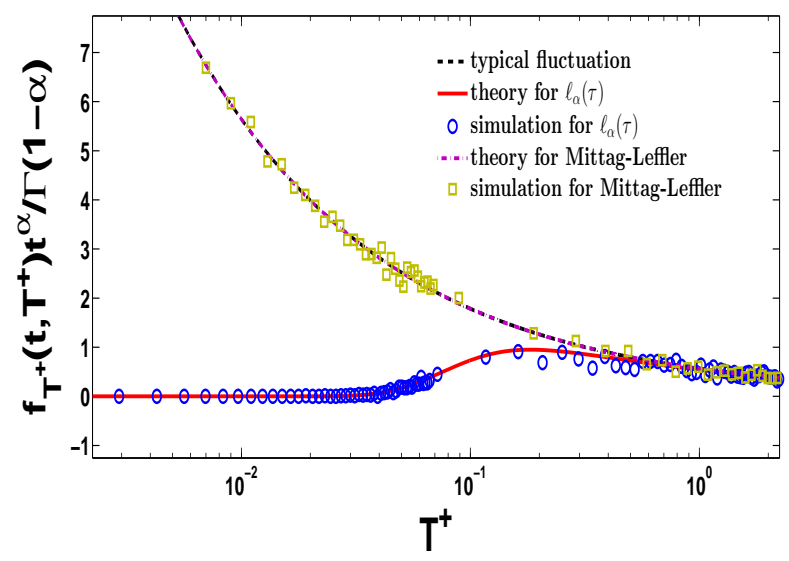

FIG. 15: (color online) The scaled PDF of the occupation time versus $T^{+}$generated by the trajectories of particles with $\alpha=0.5$ and $t=1000$ for $T^{+} \ll t$. The solid (red) line and the dash-dot line correspond to the theoretical results given by Eqs. (77) and (75), respectively, depicting the large deviations with $T^{+} \propto t^{0}$. The dashed (black) line, given by Eq. (72), shows the typical fluctuations. Note that it overlaps with theoretical result of Mittag-Leffler waiting time, the top curve in the figure.

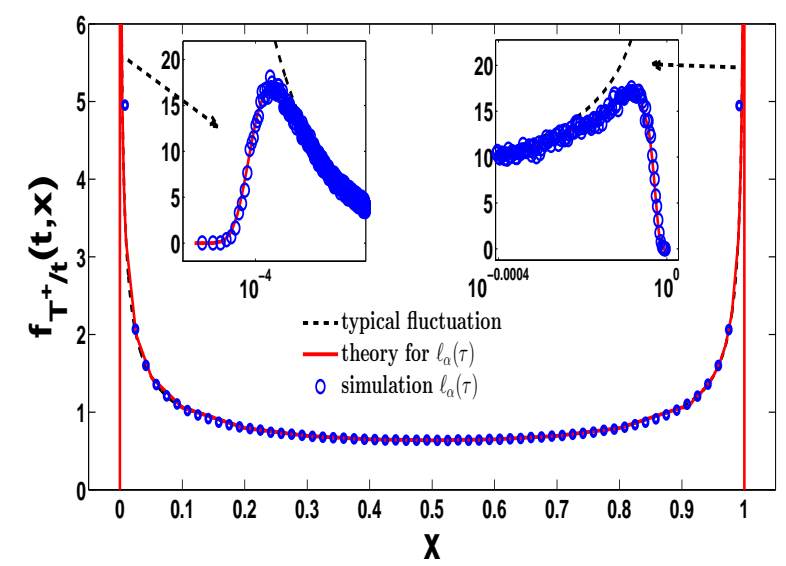

FIG. 16: (color online) The occupation time density $f_{T^{+} / t}(t, x)$ of a renewal process with $\phi(\tau)$ Eq. (6). The parameters are $\alpha=0.5$ and $t=1000$. The simulations, plotted by the symbols, are generated by averaging $10^{7}$ trajectories and the curve is the theoretical result obtained from Eq. (77) and symmetry of $f_{T^{+}}(t, x)$. Note that the results for typical fluctuations Eq. (72), diverge on $x=0$ and $x=1$, while the large deviations theory predicts correctly finite value of the PDF.

Laplace transform, yields the PDF of $T^{+} / t[1,51]$

$$
\begin{aligned}
\lim _{t \rightarrow \infty} & f_{T^{+} / t}(x) \sim \frac{\sin (\pi \alpha)}{\pi} \\
& \times \frac{x^{\alpha-1}(1-x)^{\alpha-1}}{x^{2 \alpha}+(1-x)^{2 \alpha}+2 \cos (\pi \alpha) x^{\alpha}(1-x)^{\alpha}}
\end{aligned}
$$

with $0<x<1$. It implies that the probability distribution of the random variable $x=T^{+} / t$ will converge in the limit of long $t$, to a limiting distribution which is $t$ independent. In the particular case $\alpha=1 / 2$, Eq. (71) reduces to the arcsine law on $[0,1]$

$$
\lim _{t \rightarrow \infty} f_{T^{+} / t}(x) \sim \frac{1}{\pi \sqrt{x(1-x)}}
$$

or

$$
f_{T^{+}}\left(t, T^{+}\right)=\frac{1}{\pi \sqrt{T^{+}\left(t-T^{+}\right)}} .
$$

Eq. (71) was originally derived by Lamperti [51]; see also Darling-Kac law [39]. The typical fluctuations described by Eq. (72) is plotted by the dashed (black) lines; see Figs. 15 and 16. Besides, for $\alpha=0.5$ the typical result Eq. (72) implies that $f_{T^{+} / t}(x)$ blows up when $x \rightarrow 0$ and $x \rightarrow 1$.

Next we analyse the case of $T^{+} \ll t$, i.e., $s \ll u$. Based on Eq. (70), we find the infinite density

$$
f_{T^{+}}\left(t, T^{+}\right) \sim \mathcal{L}_{T^{+}}^{-1}\left[-\frac{1}{2}+\frac{1}{1-\widehat{\phi}(u)}\right] p_{0}(t),
$$

where $p_{0}(t)$ is the survival probability defined by Eq. (10). Note that Eq. (74) is not normalised, which is not a problem since it is valid for $T^{+} \ll t$.

We now investigate the infinite density Eq. (74) with two choices of $\phi(\tau)$. Similar to our previous examples the infinite density depends on the spectfics of $\phi(\tau)$ unlike the Lamperti law Eq. (71). Using the example of a MittagLeffler PDF $\phi(\tau)$, pluging Eq. (29) into Eq. (74) and then taking the inverse Laplace transform

$$
f_{T^{+}}\left(t, T^{+}\right) / p_{0}(t) \sim \frac{1}{2} \delta\left(T^{+}\right)+\frac{1}{\Gamma(\alpha)}\left(T^{+}\right)^{\alpha-1}
$$

see Fig. 15. The first term on the right hand side is a delta function, it describes events where the process starts at state minus and remains there for time $t$ (the factor $1 / 2$ is due to the initial condition, the probability of $1 / 2$ to start in the state up or down). Furthermore, it is interesting to find that the typical result Eq. (71) is consistent with the theoretical result with Mittag-Leffler time statistics for all kinds of $0<T^{+} \ll t$, not including the delta function in Eq. (75).

Comparing Eq. (75) with typical fluctuations Eq. (73), we observe that for $\alpha=1 / 2$ the occupation time with Mittag-Leffler waiting time produces large deviations statistics that are very similar to typical events statistics. But even in this as close as can get scenario, we find an feature being exclusively revealed by the large deviations analysis. Namely, there is a discrete probability to find the occupation time being trapped in an initial state.

Now we derive a formal solution for the rare events. Using the relation $1 /(1-\widehat{\phi}(u))=\sum_{n=0}^{\infty} \widehat{\phi}^{n}(u)$ and performing the double inverse Laplace transform, leading to

$$
f_{T^{+}}\left(t, T^{+}\right) \sim\left(\frac{1}{2} \delta\left(T^{+}\right)+\sum_{n=1}^{\infty} \mathcal{L}_{T^{+}}^{-1}\left[\widehat{\phi}^{n}(u)\right]\right) p_{0}(t) .
$$


When $\phi(\tau)$ is one sided Lévy stable distribution, Eq. (76) reduces to

$$
f_{T^{+}}\left(t, T^{+}\right) \sim\left(\frac{1}{2} \delta\left(T^{+}\right)+\sum_{n=1}^{\infty}\left(\frac{1}{n^{1 / \alpha}} \ell_{\alpha}\left(\frac{T^{+}}{n^{1 / \alpha}}\right)\right)\right) p_{0}(t) .
$$

It can be noticed that the behavior of $f_{T^{+}}\left(t, T^{+}\right)$is determined by the shape of $\phi(\tau)$ for small $T^{+}$. Eq. (77), or more precisely the limit $t \rightarrow \infty$ of $f_{T^{+}}\left(t, T^{+}\right) / p_{0}(t)$, is the infinite density describing the occupation time statistics when $\phi(\tau)$ is the one sided Lévy distribution, see Fig. 15 for illustration.

Now we investigate the total probability to find $0<$ $T^{+}<T_{1}^{+}$, defined by $P\left(T_{1}^{+}\right)=\int_{0}^{T_{1}^{+}} f_{T^{+}}\left(t, T^{+}\right) d T^{+}$. To simplify the discussion, we just consider Mittag-Leffler time statistics. Using Eq. (75) and the asymptotic behaviors of $t^{\alpha-1} E_{\alpha, \alpha}\left(-t^{\alpha}\right)$ yields

$$
P\left(T_{1}^{+}\right) \sim \frac{1}{2} p_{0}(t)+\frac{\sin (\pi \alpha)}{\pi \alpha} \frac{\left(T_{1}^{+}\right)^{\alpha}}{t^{\alpha}}
$$

with $T_{1}^{+} \ll t$. On the other hand, in the particular case $\alpha=1 / 2$, utilizing the typical fluctuations Eq. (73) gives the arcsine distribution

$$
P\left(T_{1}^{+}\right) \sim \frac{2}{\pi} \arcsin \left(\frac{T_{1}^{+}}{\sqrt{t T_{1}^{+}}}\right)
$$

It can be noted that Eq. (79) reduces to $2 \sqrt{T_{1}^{+}} /(\pi \sqrt{t})$ for $T_{1}^{+} \ll t$. In this case, we see that Eqs. (78) and (79) are consistent with each other except for the the first term of Eq. (78). It implies that, though Eq. (74) is not normaized, we can use it to calculate some observables.

\section{B. Occupation time with $1<\alpha<2$}

Now we study the random variable $\epsilon=T^{+}-t / 2$, shifting the symmetry axis of $f_{T^{+}}\left(t, T^{+}\right)$to zero. Similar to the derivation of Eq. (45), the double Laplace transform of $f_{\epsilon}(t, \epsilon)$ is

$$
\begin{aligned}
\widehat{f}_{\epsilon}\left(s, u_{\epsilon}\right)= & \left(s+\frac{u_{\epsilon}\left(\widehat{\phi}\left(s+\frac{u_{\epsilon}}{2}\right)-\widehat{\phi}\left(s-\frac{u_{\epsilon}}{2}\right)\right)}{1-\widehat{\phi}\left(s+\frac{u_{\epsilon}}{2}\right) \widehat{\phi}\left(s-\frac{u_{\epsilon}}{2}\right)}\right) \\
& \times \frac{1}{\left(s-\frac{u_{\epsilon}}{2}\right)\left(s+\frac{u_{\epsilon}}{2}\right)} .
\end{aligned}
$$

Since the sign of $\epsilon$ is not fixed, i.e., it can be positive or negative, we replace $u_{\epsilon}$ with $-i k$ and move to the Fourier space. For the typical case, i.e., $|\epsilon| \sim t^{1 / \alpha}$, we find

$$
\widehat{f}_{\epsilon}(s, k) \sim \frac{1}{s-\frac{b_{\alpha}}{2\langle\tau\rangle}\left(\left(s+\frac{i k}{2}\right)^{\alpha}+\left(s-\frac{i k}{2}\right)^{\alpha}\right)} .
$$

Taking inverse Laplace and Fourier transform yields [34]

$$
f_{\epsilon}(t, \epsilon) \sim\left\{\begin{array}{lr}
\frac{C_{o c c}}{t^{1 / \alpha}} L_{\alpha}\left(\frac{C_{o c c}}{t^{1 / \alpha}}|\epsilon|\right), & \text { for }-t / 2<\epsilon<t / 2 \\
0, & \text { otherwise }
\end{array}\right.
$$

where $C_{o c c}=\langle\tau\rangle^{1 / \alpha} /\left(2\left(b_{\alpha}|\cos (\pi \alpha / 2)|\right)^{1 / \alpha}\right)$ and $L_{\alpha}(x)$ denotes the symmetric stable Lévy Law with the index of $\alpha$, so the Fourier transform of $L_{\alpha}(x)$ is $\exp \left(-|k|^{\alpha}\right)$, which is a special case of $L_{\alpha, \beta}(x)$; see Appendix C.

Since $0<T^{+}<t$, we find that $-t / 2<\epsilon<t / 2$. It means that the order of $\epsilon$ can be as large as the observation time $t$. Hence to investigate the rare events, we consider $s$ is of the order of $|k|$. By inverting the Fourier and Laplace transform, we find (see also [34])

$$
f_{\epsilon}(t, \epsilon) \sim \frac{\alpha b_{\alpha}}{t^{\alpha}|\Gamma(1-\alpha)|} \times\left\{\begin{array}{lr}
\chi\left(\frac{2|\epsilon|}{t}\right), & -\frac{t}{2}<\epsilon<\frac{t}{2} ; \\
0, & \text { otherwise }
\end{array}\right.
$$

with

$$
\chi(z)=\theta(0<z \leq 1) z^{-1-\alpha}\left(1-\frac{\alpha-1}{\alpha} z\right) .
$$

We see that $t^{\alpha} f_{\epsilon}(t, \epsilon)$ does not depend on the exact shape of $\phi(\tau)$ besides the parameters $\alpha$ and $b_{\alpha}$. Further the integral of Eq. (83) with respect to $\epsilon$, in the range $\epsilon \rightarrow 0$, is divergent. Thus, $f_{\epsilon}(t, \epsilon)$ is a non-normalized solution since its behavior, at $T^{+} \rightarrow t / 2$, is non-integrable. See the discussions and numerical examples in Ref. [34].

\section{DISCUSSION}

It is well known that when the averaged time interval between renewal events diverges, i.e., $0<\alpha<1$, the typical scale of the process is the measurement time and so observables of interest scale with $t$. Hence the rare fluctuations, and the far tails of the distributions of observables considered in this paper, have corrections when the observable is of the order of $t^{0}$. This leads to nonnormalized states which describe these rare events. The opposite takes place when $1<\alpha<2$ namely when the mean sojourn time is finite but the variance is diverging. Here, we have a finite scale, but when observables like $B, F, Z$ or $T^{+}$become large, namely when they are of the order of $t$, one naturally finds deviations from typical laws. Since the approximation in the far tail of the distribution must match the typical fluctuations which are described by fat tailed densities, we get by extension non-normalized states.

The uniform approximation provided in the text (for example Eq. (54) and the corresponding Fig. 11) bridges between the typical and rare fluctuations. It is obtained by matching the far tail distribution with its bulk fluctuations. Technically we find unifrom approximation by using exact theoretical results (see Eq. 34), an approximation where we take $s \rightarrow 0$ (meaning $t \rightarrow \infty$ ) leaving 
the second variable $u$ (corresponding for example to $\mathrm{F}$ ) finite (see example Eq. (37)), and for special choice of the waiting time PDF we can get the solution in terms of infinite sums (for instance Eq. (62)). In principle the uniform approximation can be used to calculate quantifiers of the process like moments. However, it is much simpler to classify observables based on their integrability with respect to the non-normalized state, as is done in infinite ergodic theory. In the case of integrable observables, we may use the non-normalized state for the calculation of integrable expectations, some what similar to the calculation averages observables from normalized densities.

Importantly, the non-normalized states are not only a tool with which we obtain moments. As we have demonstrated both theoretically and numerically, they describe the perfectly normalized probability density of the observables, when the latter are properly scaled with time (see Fig. 5). Maybe the main achievement of this paper is that we have obtained explicit solutions describing the rare events and this we did with relatively simple tools. It is rewarding that while the rare fluctuations are nonuniversal, in the sense that they depend on the details of the waiting time PDF, they can be obtained rather generally. Further, as we have shown for the backward and forward recurrence time, the density describing the typical fluctuations for $1<\alpha<2$ Eq. (36), describes the non-typical events for $0<\alpha<1$, all we need to do is replace the finite mean waiting time with an effective time dependent one; see Eq. (31).

As mentioned in the introduction the distribution of the occupation time for Brownian motion and random walks is the arcsine law, and the same holds for the backward recurrence time (here $\alpha=1 / 2$ ). While analyzing the rare events of these well known results, we see that the large deviations for these two observables behave differently (compare Fig. 9 with Fig. 16). For the backward recurrence time $B$ we have deviations from arcsine law which differ for the case $B \propto t^{0}$ and $B \propto t$ (see Fig. 9). The same symmetry breaking is not found for the occupation time since by construction of the model the probability to be in the up (+) and down (-) state is the same (Fig. 16). It should be noted that we have worked all along with a non-equilibrium process, in the sense that the process started at time $t=0$. Further, for occupations time we assumed that initially we are either in the up or down states with equal probability. In an ongoing process, when the observation starts long after the start of the process, our results for the rare and even for the typical fluctuations must be modified. For example if we start the process at time $-t_{a}$ before the process is observed at time $t=0$ we expect aging effects when $0<\alpha<1$. The effect of initial preparation on the rare fluctuations is left for a future work, as well as the connection of results presented here with the big jump principle [17].

\section{Acknowledgments}

The support of Israel Science Foundation's grant $1898 / 17$ is acknowledged, and the work was partially supported by the National Natural Science Foundation of China under Grant No. 11671182, and the Fundamental Research Funds for the Central Universities under Grant No. lzujbky-2018-ot03 and lzujbky-2017-it57. W.W. is sustained by the China Scholarship Council (CSC).

\section{Appendix A: Generation of Random Variables}

When generating the random variables with the PDF $\ell_{\alpha}(\xi)$ or $\xi^{\alpha-1} E_{\alpha, \alpha}\left(-\xi^{\alpha}\right)$ needed to simulate the renewal process, the Monte Carlo statistical methods [52] are used. Chambers et al. [53] showed how to obtain a random variable drawn from the stable Lévy distribution with $0<\alpha<1$. Furthermore, Kozubowski constructed the following structural representation of a $\phi(\xi)=\xi^{\alpha-1} E_{\alpha, \alpha}\left(-\xi^{\alpha}\right)$ distributed random variable $\xi$ as [38]

$$
\xi=\sigma \eta^{1 / \alpha}
$$

where $\sigma$ is a random number from the exponential distribution with mean parameter 1 , and $\eta$ has the PDF

$$
f(\eta)=\frac{\sin (\pi \alpha)}{\alpha \pi\left(\eta^{2}+2 \eta \cos (\pi \alpha)+1\right)}
$$

with $0<\alpha<1$ and $\eta>0$.

\section{Appendix B: The derivation of some important formulas}

Now we give a brief account on some main equations used in the paper. Let us consider a process starting at time $t=0$ and define $Q_{N}(t)$, which is the probability that the $N$-th event happens at time $t=t_{N}=$ $\tau_{1}+\tau_{2}+, \ldots,+\tau_{N}$ (see Fig. 1). Note that an important relation between $Q_{N}(t)$ and $Q_{N-1}(t)$ is Eq. (9). Then using the convolution theorem of Laplace transform and $Q_{1}(\tau)=\phi(\tau)$, it follows that

$$
\widehat{Q}_{N}(s)=\widehat{\phi}^{N}(s) .
$$

Another important equality is the conditional probability density of the forward recurrence time $F$ given that exactly $N$ events occurred before time $t$, defined by

$$
f_{N}(t, F)=\int_{0}^{t} Q_{N}(\tau) \phi(t-\tau+F) d \tau
$$

Note that the forward recurrence time is given by $f_{F}(t, F)=\sum_{N=0}^{\infty} f_{N}(t, F)$, i.e., Eq. (25). The Laplace 
transform of $f_{F}(t, F)$ with respect to $t$ follows from the shift theorem of Laplace transform and reads

$$
\widehat{f}_{F}(s, F)=\frac{1}{1-\widehat{\phi}(s)} \exp (s F) \int_{F}^{\infty} \phi(z) \exp (-s z) d z .
$$

Then, taking Laplace transform and using partial integration, lead to the final result Eq. (26).

Now, our aim is to obtain the PDF of the occupation time. Let $Q_{N}\left(t, T^{+}\right)$be the PDF of the occupation time just arriving at $T^{+}$at time $t$ after finishing $N$ steps. $Q_{N}^{ \pm}\left(t, T^{+}\right)$is

$$
\begin{aligned}
Q_{N+2}^{+}\left(t, T^{+}\right) & =\int_{0}^{T^{+}} \int_{0}^{t} Q_{N}\left(t-\tau-z, T^{+}-z\right) \\
& \times \phi(z) \phi(\tau) d \tau d z+q \delta(t) \delta\left(t-T^{+}\right)
\end{aligned}
$$

and

$$
\begin{aligned}
Q_{N+2}^{-}\left(t, T^{+}\right) & =\int_{0}^{T^{+}} \int_{0}^{t} Q_{N}\left(t-\tau-z, T^{+}-z\right) \\
& \times \phi(z) \phi(\tau) d \tau d z+(1-q) \delta(t) \delta\left(T^{+}\right),
\end{aligned}
$$

where \pm in the superscript of $Q_{N+2}^{ \pm}\left(t, T^{+}\right)$means that the initial state of the particle is \pm . $q$ is the probability that the initial state is + , with $0 \leq q \leq 1$. In double Laplace space, representation of the above Eq. (B3) takes an especially simple form

$$
\widehat{Q}_{N+2}^{+}(s, u)=\frac{q}{1-\widehat{\phi}(s+u) \widehat{\phi}(s)} .
$$

Then the $\mathrm{PDF} f_{T^{+}}\left(t, T^{+}\right)$is

$$
\begin{aligned}
& f_{T^{+}}\left(t, T^{+}\right)=\sum_{N=0}^{\infty}( \\
& \int_{0}^{t} \int_{0}^{T^{+}} Q_{N}^{+}\left(t-\tau-z, T^{+}-z\right) \int_{z}^{\infty} \phi(y) d y d z d t \\
& +\int_{0}^{t} \int_{0}^{T^{+}} Q_{N}^{+}\left(t-\tau-z, T^{+}-z\right) \phi(z) \int_{\tau}^{\infty} \phi(y) d y d z d t \\
& +\int_{0}^{t} \int_{0}^{T^{+}} Q_{N}^{-}\left(t-\tau-z, T^{+}-z\right) \phi(\tau) \int_{z}^{\infty} \phi(y) d y d z d t \\
& \left.+\int_{0}^{t} Q_{N}^{-}\left(t-\tau, T^{+}\right) \int_{\tau}^{\infty} \phi(y) d y d t\right) .
\end{aligned}
$$

Taking double Laplace transform, summing the infinite terms, and then from Eq. (B5) it follows that

$$
\begin{aligned}
\widehat{f}_{T^{+}}(s, u)= & \frac{u(1-q-q \widehat{\phi}(s+u))(1-\widehat{\phi}(s))}{s(s+u)(1-\widehat{\phi}(s) \widehat{\phi}(s+u))} \\
& +\frac{s}{s(s+u)} .
\end{aligned}
$$

For $q=1 / 2$, Eq. (B6) reduces to Eq. (70) and the corresponding typical fluctuations are studied in Refs. [1, 28].

\section{Appendix C: Some properties of Stable distribution}

Now we discuss the series representation and the asymptotic behavior of stable distribution $L_{\alpha, \beta}(x)$ [10, $26,54]$. The corresponding PDF $L_{\alpha, \beta}(x)$ is given by the inverse Fourier transform

$L_{\alpha, \beta}(x)=\frac{1}{2 \pi} \int_{0}^{\infty} \exp \left(-i k x-c|k|^{\alpha}\left(1+i \beta \frac{z}{|z|} h(z, \alpha)\right)\right) d k$,

where $\alpha, \beta, c$ are constants and

$$
h(z, \alpha)= \begin{cases}\tan \left(\frac{\pi \alpha}{2}\right), & \alpha \neq 1 ; \\ \frac{\pi}{2} \log (z), & \alpha=1 .\end{cases}
$$

Especially, for $\beta=0$ and $c=1$, Eq. (C1) reduces to the symmetric stable distribution $L_{\alpha}(x)$. For simplification of analysis, let $\beta=1, \alpha \neq 1$, and $c=-\cos (\pi \alpha / 2)$,

$$
L_{\alpha, 1}(x)=\frac{1}{2 \pi} \int_{-\infty}^{\infty} \exp (-i k x) \exp \left[(i k)^{\alpha}\right] d k .
$$

Expanding the integrand in the right hand side as a Taylor series in $x$ yields the convergent series

$$
\begin{aligned}
L_{\alpha, 1}(x) & =\sum_{n=0}^{\infty} \frac{(-1)^{n}}{\alpha \pi(2 n) !} \Gamma\left(\frac{2 n+1}{\alpha}\right) \cos (g(n, \alpha)) x^{2 n} \\
& -\sum_{n=0}^{\infty} \frac{\operatorname{sign}(x)(-1)^{n} \Gamma\left(\frac{2 n+2}{\alpha}\right)}{\pi \alpha(2 n+1) !} \\
& \times \sin \left(g\left(n+\frac{1}{2}, \alpha\right)\right) x^{2 n+1},
\end{aligned}
$$

where $g(n, \alpha)=(2 n+1) \pi(1 / 2-1 / \alpha)$ and $\operatorname{sign}(x)=x /|x|$ for $|x|>0$ and zero otherwise. Especially, for $x \rightarrow 0$, Eq. (C2) reduces to

$$
L_{\alpha, 1}(x) \sim \frac{\Gamma\left(\frac{1}{\alpha}\right)}{\alpha \pi} \cos (g(0, \alpha)),
$$

which is a constant and strictly less than $L_{\alpha}(0)$ for $\alpha<2$. Furthermore, the asymptotic behavior of $L_{\alpha, 1}(x)$ is

$$
L_{\alpha, 1}(x) \sim \sum_{n=1}^{\infty} \frac{\Gamma(1+\alpha n) \sin (\alpha n \pi)}{2 \pi n !|x|^{1+\alpha n}}(-1+\operatorname{sign}(x)),
$$

it implies that $L_{\alpha, 1}(x) \sim|x|^{-1-\alpha} / \Gamma(-\alpha)$, being the same as the left hand side of the tail of the symmetric Lévy stable distribution, for $x \rightarrow-\infty$ and the tails of $L_{\alpha, 1}(x)$ are asymmetric with respect to $x$.

\section{Appendix D: The calculation of $q$ order moments for forward recurrence time with $\alpha>1$}

Using the calculated results of $f_{F}(t, F)$, we study fractional moments $\left\langle F^{q}\right\rangle$. First, we obtain the low order 
moments with $\alpha>1$, i.e., $q<\alpha-1$. Using Eqs. (36) and (39), and utilizing integration by parts

$$
\left\langle F^{q}\right\rangle \sim \frac{1}{(q+1)\langle\tau\rangle} \int_{0}^{\infty} F^{q+1} \phi(F) d F .
$$

We notice that the right hand side of Eq. (D1) is a finite number due to $q-\alpha<-1$. Then, we discuss the case of $q>\alpha-1$. According to Eq. (38)

$$
\begin{aligned}
\left\langle F^{q}\right\rangle \sim & \lim _{z \rightarrow 0} \int_{z}^{\infty}\left(F^{-\alpha}-(F+t)^{-\alpha}\right) \frac{b_{\alpha} F^{q}}{|\Gamma(1-\alpha)|\langle\tau\rangle} d F \\
\sim & \lim _{z \rightarrow 0} \frac{b_{\alpha} z^{1-\alpha+q}}{|\Gamma(1-\alpha)|\langle\tau\rangle(1+q-\alpha)} \\
& \times F\left(\alpha,-1+\alpha-q, \alpha-q ;-\frac{t}{z}\right)
\end{aligned}
$$

where $F(\alpha, \beta, \gamma ; x)$ is the hypergeometric function [55, 56], defined by

$$
F(\alpha, \beta, \gamma ; x)=1+\sum_{n=1}^{\infty} \frac{(\alpha)_{n}(\beta)_{n}}{(\gamma)_{n}} \frac{x^{n}}{n !}
$$

with $(\alpha)_{n}=\alpha(\alpha+1) \ldots(\alpha+k-1)$. Note that the asymptotic behavior of $F(\alpha, \beta, \gamma ;-x)$ is

$$
F(\alpha, \beta, \gamma ;-x) \sim x^{-\alpha} \frac{\Gamma(\beta-\alpha) \Gamma(\gamma)}{\Gamma(\beta) \Gamma(\gamma-\alpha)}+x^{-\beta} \frac{\Gamma(\alpha-\beta) \Gamma(\gamma)}{\Gamma(\alpha) \Gamma(\gamma-\beta)}
$$

with $x>0$. Using Eq. (D4), the dominant term of Eq. (D2) gives

$$
\left\langle F^{q}\right\rangle \sim \frac{b_{\alpha} \Gamma(1+q) \Gamma(\alpha-q)}{|\Gamma(1-\alpha)|\langle\tau\rangle(1+q-\alpha) \Gamma(\alpha)} t^{1-\alpha+q},
$$

keep in mind that substituting uniform approximation Eq. (37) into Eq. (39) yields the same result as Eq. (D5). In the particular case $q=1$, we have $\langle F\rangle \sim t^{2-\alpha}$. Besides, when $q>\alpha$, as expected $\left\langle F^{q}\right\rangle$ diverges.

\section{Appendix E: $q$ order moments with $\alpha<1$}

We now study the fractional moments of $B$. Note that $B^{q}$ with $q>\alpha$, are non-integrable with respect to the non-normalized density Eq. (43). We find that the fractional moments of $B$ are governed by the typical fluctuations Eq. (50), namely

$$
\begin{aligned}
\left\langle B^{q}\right\rangle & =\int_{0}^{\infty} B^{q} f_{B}(B, t) d B \\
& \sim \frac{\sin (\pi \alpha) \Gamma(\alpha) \Gamma(1-\alpha+q)}{\pi \Gamma(1+q)} t^{q} .
\end{aligned}
$$

We check this result in the following: for a natural number $q$, expanding Eq. (41) as a Taylor series in $u$, and performing the inverse Laplace transform term by term, we obtain the corresponding moments, which are the same as Eq. (E1).

Let us consider another interesting observable, i.e., the moments of $N$. Using Eqs. (14) and (39)

$$
\begin{aligned}
\left\langle N^{q}\right\rangle & \sim \int_{0}^{\infty} \frac{t N^{q}}{\alpha N^{1+1 / \alpha} b_{\alpha}^{1 / \alpha}} \ell_{\alpha}\left(\frac{t}{\left(N b_{\alpha}\right)^{1 / \alpha}}\right) d N \\
& =\frac{\int_{0}^{\infty} \xi^{-\alpha q} \ell_{\alpha}(\xi) d \xi}{\left(b_{\alpha}\right)^{q}} t^{\alpha q}
\end{aligned}
$$

In the particular case $q \rightarrow 0$, the normalized condition is found, namely, $\left\langle N^{0}\right\rangle=1$.

To summarize, if $\alpha<1$, then for all observables in this paper, i.e., $N, F, B, Z$, and $T^{+}$, the moments (if they exist) are obtained by the PDF describing the typical fluctuations. Note that for $F$ and $Z$, high order $(q>$ $\alpha)$ moments diverge. One may wonder in what sense is Eq. (43) an infinite density? For that we consider the observable $\Theta\left(B_{1}<B<B_{2}\right)$ with $B_{1}, B_{2} \ll t$, where $\Theta\left(B_{1}<B<B_{2}\right)$ is one if the condition holds. Then

$$
\begin{aligned}
\left\langle\Theta\left(B_{1}<B<B_{2}\right)\right\rangle & =\int_{0}^{\infty} \Theta\left(B_{1}<B<B_{2}\right) f_{B}(t, B) d B \\
& \sim \frac{1}{\left\langle\tau^{*}\right\rangle} \int_{B_{1}}^{B_{2}} \int_{B}^{\infty} \phi(y) d y d B
\end{aligned}
$$

where $\left\langle\tau^{*}\right\rangle$, defined below Eq. (31), is the effective average waiting time. In other words, the observable $\Theta\left(B_{1}<B<\theta_{2}\right)$ is integrable with respect to the nonnormalized density, and hence the latter is used for the calculation of the average $\Theta\left(B_{1}<B<\theta_{2}\right)$.
[1] C. Godrèche and J. M. Luck, J. Stat. Phys. 104, 489 (2001).

[2] F. Mainardi, R. Gorenflo, and E. Scalas, Vietnam J. Math. 32, 53 (2004).

[3] F. Mainardi, R. Gorenflo, and A. Vivoli, J. Comput. Appl. Math. 205, 725 (2007).

[4] C. Godrèche, S. N. Majumdar, and G. Schehr, J. Stat. Mech: Theory Exp. 2015, P03014 (2015).

[5] M. Niemann, E. Barkai, and H. Kantz, Math. Model.
Nat. Phenom. 11, 191 (2016).

[6] T. Miyaguchi, T. Akimoto, and E. Yamamoto, Phys. Rev. E 94, 012109 (2016).

[7] S. Bianco, P. Grigolini, and P. Paradisi, J. Chem. Phys. 123, 174704 (2005).

[8] Y. Edery, S. Berg, and D. Weitz, Phys. Rev. Lett. 120, 028005 (2018).

[9] A. Weron, K. Burnecki, E. J. Akin, L. Solé, M. Balcerek, M. M. Tamkun, and D. Krapf, Sci. Rep. 7, 5404 (2017). 
[10] R. Metzler and J. Klafter, Phys. Rep. 339, 1 (2000).

[11] E. Barkai, E. Aghion, and D. A. Kessler, Phys. Rev. X 4, 021036 (2014).

[12] S. Boettcher, D. M. Robe, and P. Sibani, arXiv:1803.06580 (2018).

[13] M. Nyberg, L. Lizana, and T. Ambjörnsson, Phys. Rev. E 97, 032114 (2018).

[14] A. A. Fernández-Marín, J. A. Méndez-Bermúdez, J. Carbonell, F. Cervera, J. Sánchez-Dehesa, and V. A. Gopar, Phys. Rev. Lett. 113, 233901 (2014).

[15] H. Touchette, Phys. Rep. 478, 1 (2009).

[16] S. Whitelam, Phys. Rev. E 97, 062109 (2018).

[17] A. Vezzani, E. Barkai, and R. Burioni, arXiv:1804.02932 (2018).

[18] A. Rebenshtok, S. Denisov, P. Hänggi, and E. Barkai, Phys. Rev. E 90, 062135 (2014).

[19] E. Aghion, D. A. Kessler, and E. Barkai, Phys. Rev. Lett. 118, 260601 (2017).

[20] E. Aghion, D. A. Kessler, and E. Barkai, arXiv:1804.05571 (2018).

[21] J. Aaronson, An introduction to infinite ergodic theory, vol. 50 of Mathematical Surveys and Monographs (American Mathematical Society, Providence, RI, 1997).

[22] S. Redner, A Guide to First-Passage Processes (Cambridge University Press, Cambridge, 2001).

[23] P. Mörters and Y. Peres, Brownian Motion, vol. 30 of Cambridge Series in Statistical and Probabilistic Mathematics (Cambridge University Press, Cambridge, 2010).

[24] T. Akimoto and E. Yamamoto, Phys. Rev. E 93, 062109 (2016).

[25] T. Sadhu, M. Delorme, and K. J. Wiese, Phys. Rev. Lett. 120, 040603 (2018).

[26] R. Metzler and J. Klafter, J. Phys. A: Math. Gen. 37, R161 (2004).

[27] X. Brokmann, J.-P. Hermier, G. Messin, P. Desbiolles, J.-P. Bouchaud, and M. Dahan, Phys. Rev. Lett. 90, 120601 (2003).

[28] G. Margolin, V. Protasenko, M. Kuno, and E. Barkai, Power-Law Blinking Quantum Dots: Stochastic and Physical Models, chap. 4, 327-356 (Wiley-Blackwell, 2005).

[29] W. Feller, An introduction to probability theory and its applications. Vol. II. Second edition (John Wiley \& Sons, Inc., New York, 1971).

[30] S. Burov and E. Barkai, Phys. Rev. E 86, 041137 (2012).

[31] E. W. Montroll and G. H. Weiss, J. Math. Phys. 6, 167 (1965).

[32] J. H. P. Schulz, E. Barkai, and R. Metzler, Phys. Rev. X 4, 011028 (2014).

[33] I. Podlubny, Fractional Differential Equations (Academic Press, Inc., San Diego, 1999).
[34] J. H. P. Schulz and E. Barkai, Phys. Rev. E 91, 062129 (2015).

[35] E. B. Dynkin, Selected Translations in Mathematical Statistics and Probability (American Mathematical Society, Providence, 1961).

[36] R. Kutner and J. Masoliver, Eur. Phys. J. B 90, 50 (2017)

[37] K. A. Takeuchi and T. Akimoto, J. Stat. Phys. 164, 1167 (2016).

[38] T. J. Kozubowski, Math. Comput. Modelling 34, 1023 (2001).

[39] D. A. Darling and M. Kac, Trans. Amer. Math. Soc. 84, 444 (1957).

[40] K. K. Jose, P. Uma, V. S. Lekshmi, and H. J. Haubold, In Proceedings of the Third UN/ESA/NASA Workshop on the International Heliophysical Year 2007 and Basic Space Science, Astrophys. Space Sci. Proc., 79-92 (Springer, Heidelberg, 2010).

[41] M. Thaler and R. Zweimüller, Probab. Theory Related Fields 135, 15 (2006).

[42] T. Akimoto, Phys. Rev. Lett. 108, 164101 (2012).

[43] N. Korabel and E. Barkai, Phys. Rev. Lett. 102, 050601 (2009).

[44] J. K. E. Tunaley, J. Stat. Phys. 11, 397 (1974).

[45] A. D. Polyanin and A. V. Manzhirov, Handbook of Mathematics for Engineers and Scientists (Chapman \& Hall/CRC, Boca Raton, 2007).

[46] J. Bertoin, T. Fujita, B. Roynette, and M. Yor, Probab. Math. Statist. 26, 315 (2006).

[47] K. L. Chung, Ark. Mat. 14, 155 (1976).

[48] R. K. Getoor and M. J. Sharpe, Zeitschrift für Wahrscheinlichkeitstheorie und Verwandte Gebiete 47, 83 (1979).

[49] S. N. Majumdar and A. Comtet, Phys. Rev. Lett. 89, 060601 (2002).

[50] S. N. Majumdar, Current Sci. 77, 370 (1999).

[51] J. Lamperti, Trans. Amer. Math. Soc. 88, 380 (1958).

[52] C. P. Robert and G. Casella, Monte Carlo Statistical Methods (Springer-Verlag, New York, 2004).

[53] J. M. Chambers, C. L. Mallows, and B. W. Stuck, J. Amer. Statist. Assoc. 71, 340 (1976).

[54] W. R. Schneider, Stable distributions: Fox functions representation and generalization, vol. 262 of Lecture Notes in Phys., 497-511 (Springer, Berlin, 1986).

[55] M. Abramowitz and I. A. Stegun (eds.), Handbook of Mathematical Functions with Formulas, Graphs, and Mathematical Tables (John Wiley \& Sons, Inc., New York, 1984).

[56] J. B. Seaborn, Hypergeometric Functions and Their Applications, vol. 8 (Springer-Verlag, New York, 1991). 